Pacific Journal of Mathematics

ON A CONNECTION BETWEEN NILPOTENT GROUPS AND
OSCILLATORY INTEGRALS ASSOCIATED TO 


\title{
ON A CONNECTION BETWEEN NILPOTENT GROUPS AND OSCILLATORY INTEGRALS ASSOCIATED TO SINGULARITIES
}

\author{
ROGER E. HOWE
}

The object of this paper is to demonstrate and promote some ties between the theory of harmonic analysis on nilpotent Lie groups theory and another topic the study of oscillatory integrals associated to polynomial singularities. Oscillatory integrals are tempered distributions on $\boldsymbol{R}^{n}$, defined by integration against the exponentials of (real-valued) polynomials.

Thus if $p$ is polynomial on $\boldsymbol{R}^{n}$ with real coefficients, the associated oscillatory integral is

$$
E_{p}(f)=\int_{\boldsymbol{R}^{n}} f(x) e^{i p(x)} d x
$$

where $f$ belongs to $\mathscr{S}\left(\boldsymbol{R}^{n}\right)$, the Schwartz space of $\boldsymbol{R}^{n}$, and $d x$ is Lebesgue measure. The main questions of which I am aware concerning the distributions $E_{p}$ are two.

(a) Asymptotic behavior: For $t \in \boldsymbol{R}$, how does $E_{t p}(f)$ behave as $t \rightarrow \infty$ ? In particular, what is the slowest rate of decay of $E_{t p}(f)$ ?

(b) Fourier transform: Define the Fourier transform ${ }^{\wedge}: \mathscr{S}\left(\boldsymbol{R}^{n}\right) \rightarrow$ $\mathscr{S}\left(\boldsymbol{R}^{n}\right)$ by the usual formula

$$
\widehat{f}(x)=\int_{\boldsymbol{R}^{n}} f(y) e^{-2 \pi i x \cdot y} d y
$$

where $x \cdot y$ is the usual inner product on $\boldsymbol{R}^{n}$. Define $\hat{E}_{p}$ by

$$
\hat{E}_{p}(f)=\int_{\boldsymbol{R}^{n}} \hat{f}(-x) e^{i p(x)} d x \text {. }
$$

Can $\hat{E}_{p}$ be represented directly as a distribution? Is it given as integration against some function? How can this function be described if it exists?

The simplest case of interest for questions (a) and (b) is that of the stable or Morse singularities, when $p$ is a nondegenerate quadratic form. Here both questions (a) and (b) have well-known, satisfying, classical answers [5]. We recall the formulas in one-dimension.

$$
\left(e^{\pi i t x^{2}}\right)^{\wedge}=\left(\frac{1+i}{\sqrt{2 t}}\right) e^{-\pi i x^{2} / t}, \quad t>0
$$




$$
\int_{-\infty}^{\infty} f(x) e^{\pi i t x^{2}} \stackrel{t \rightarrow \infty}{\sim} \frac{1+i}{\sqrt{2}} \sum_{n=0}^{\infty}\left(\frac{i}{4 \pi}\right)^{n} \frac{1}{n !} \frac{d^{2 n} f(0)}{d x^{2 n}} t^{-n-1 / 2} .
$$

It turns out that these formulas have very beautiful, I am even tempted to say deep, interpretations in terms of harmonic analysis on the Heisenberg group. We give the essence of this interpretation in $\S 4$ (see Theorem 4.3). The purpose of this paper is to investigate the extent to which the general features of the group theoretic interpretations of (4) and (5) apply also to higher order singularities.

Thus one goal of this paper is to show that both questions (a) and (b) can be embedded into the theory of harmonic analysis on nilpotent groups. The first question is related to the behavior of smooth matrix coefficients of irreducible representations, while the second has to do with the variety of ways to realize a given representation as an induced representation. A second goal of course is to develop some of the relevant nilpotent harmonic analysis. This development follows two main themes. On the one hand, we study what might be termed the smooth harmonic analysis of general nilpotent groups. This takes up some themes touched on in Kirillov's original paper [8], but neglected since. On the other hand, we isolate a class of nilpotent groups, labeled HAT groups, which are particularly relevant to the concrete problems stated above. It seems significant that in both of these investigations, groups with square integrable representations play a distinguished role.

The above described topics occupy $\S 2$ and $\S 3$. In $\S 4$ we draw some conclusions for the questions (a) and (b). The rest of this introduction describes how oscillatory integrals naturally embed into nilpotent harmonic analysis.

The point is that a multiple interpretation may be given to the exponential polynomial $e^{i p(x)}$. First, we may regard it as an unitary operator. If $f \in L^{2}\left(\boldsymbol{R}^{n}\right)$, then $U_{p}: f \rightarrow e^{i p} f$ defines an unitary transformation on $L^{2}$. We note that the Schwartz space $\mathscr{S}\left(\boldsymbol{R}^{n}\right)$ is invariant under $U_{p}$. Going slightly further, we might remark that the operators $U_{t p}$, for $t \in \boldsymbol{R}$, form a one-parameter group of unitary transformations, whose infinitesimal generator is multiplication by p. Another group of unitary operators is provided by the translations. Explicitly, for $f \in L^{2}$, put $\left(T_{y} f\right)(x)=f(x-y)$, for $x, y \in \boldsymbol{R}^{n}$. Again, the $T_{y}$ preserve $\mathscr{S}$. We now compute the conjugate of $U_{p}$ by $T_{y}$. We have

$$
\begin{aligned}
\left(T_{y}\left(U_{p}\left(T_{-y} f\right)\right)\right)(x) & =\left(U_{p}\left(T_{-y} f\right)\right)(x-y) \\
& =e^{i p(x-y)}\left(T_{-y} f\right)(x-y)=e^{i p(x-y)} f(x) .
\end{aligned}
$$

In other words, $T_{y} U_{p} T_{-y}=U_{T_{y(p)}}$. The translations do not normalize 
the one-parameter group $U_{t p}$, but they come close. Specifically, let $\mathfrak{D}_{p}=\mathfrak{D}$ be the space of polynomials on $\boldsymbol{R}^{n}$ spanned by the partial derivatives (of all orders) of $p$, and by the coordinate functions. As $y$ varies in $\boldsymbol{R}^{n}$, the polynomials $T_{y}(p)$ certainly vary inside $\mathfrak{D}_{p}$. Thus $U_{p}$ belongs to a finite-dimensional abelian group $D$ of unitary operators normalized by the translations. In particular we may take

$$
D=\left\{U_{q}: q \in \mathfrak{D}_{p}\right\} \text {. }
$$

If $A$ denotes the group of translations, we may form the semidirect product $N=A \times_{s} D$. Then $N$ is a nilpotent group of unitary operators acting on $L^{2}$. Furthermore, it is not hard to see that $N$ acts irreducibly on $L^{2}$. (This was the point of including the coordinate functions in $\mathfrak{D}_{p}$, were they not there automatically.) Still further, $N$ preserves $\mathscr{S}\left(\boldsymbol{R}^{n}\right)$, and it may be seen without much difficulty (see $\S 3$ for fuller discussion) that $\mathscr{S}$ may be described as the space of smooth vectors for $N$. Therefore (since every element of $\mathscr{S}$ is a sum of products of elements of $\mathscr{S}$ ) we see that $E_{p}(f)$ is the value at $U_{p} \in D$ of a smooth matrix coefficient (see $\S 3$ for a precise definition) of the representation of $N$ defined by the above action.

A second, related, interpretation of $E_{p}$ is as follows. It is wellknown that there is precisely one tempered distribution (up to multiples) on $R^{n}$ which is invariant under the group $A$ of translations, namely Lebesgue measure $d x$. (We will give a more precise version of this fact in $\S 4$.) Within $N$, we may conjugate the group $A$ by $U_{p}$ to obtain some other abelian subgroup $U_{p} A U_{p}^{-1}$. It is quite clear that $U_{p} A U_{p}^{-1}$ will also allow precisely one invariant distribution, which will necessarily be $E_{p}$. Together these three interpretations of $e^{i p(x)}$, as operator, as matrix coefficient, and as eigendistribution, will hopefully motivate the considerations of the next sections.

2. Structural questions. The nilpotent group $N$ constructed in $\S 1$ had various properties. For example, it was a semidirect product, it was metabelian, it had a faithful irreducible representation induced from a normal subgroup, the adjoint action on this normal subgroup was essentially cyclic, and so on. Our task in this section is to try to outline the interplay between these properties, to bring out to what extent they make $N$ special, and to describe the structure of groups having them. The statements of the results tend to be lengthy, and to avoid tedium, we have omitted most of the proofs, which are quite easy, on the whole. Modulo the Kirillov orbit theory, which we review, the discussion is completely algebraic.

We adopt the following conventions for the rest of the paper. By a nilpotent Lie group we mean a connected simply connected 
nilpotent Lie group, unless we explicitly state otherwise. Similarly, subgroups will be connected. Nilpotent Lie groups will be denoted by upper case Roman letters (not conversely!) usually in the middle of the alphabet. The Lie algebra of such a group will be denoted by the corresponding upper case German letter. Thus $N$ and $\mathfrak{R}$. We write exp for the canonical exponential map from $\mathfrak{N}$ to $N$, and $\log$ for its inverse. The adjoint action of $N$ on $\mathfrak{N}$ is written $\mathrm{Ad}$, the adjoint action of $\mathfrak{N}$ on itself is ad. Restrictions and quotients of Ad will usually still be called Ad, hopefully with sufficient specification of domains to avoid confusion. Dualizations of Ad are written as $\mathrm{Ad}^{*}$. The descending central series of $N$ is $N=N^{(1)}, N^{(2)}$, etc. The ascending central series is $\mathscr{Z}(N)$, $\mathscr{Z}^{(2)}(N)$, etc. In particular, $N^{(2)}$ is the commutator subgroup and $\mathscr{L}(N)$ the center of $N$. The notation for $\mathfrak{R}$ is parallel.

We now recall the basics of Kirillov's orbital theory of representations [8]. For a nilpotent Lie group $N$, let $\hat{N}$ be the unitary dual of $N$, the set of equivalence classes of unitary irreducible continuous representations of $N$. According to Kirillov, we may identify $\hat{N}$ with the space of $\mathrm{Ad}^{*} N$ orbits in $\mathfrak{R}^{*}$. The identification proceeds as follows. Let $\mathcal{O} \subseteq \mathfrak{N}^{*}$ be an $\mathrm{Ad}^{*} N$ orbit, and choose $\lambda \in \mathcal{O}$. Consider the alternating bilinear form $B_{\lambda}(x, y)=\lambda([x, y])$ on $\mathfrak{N}$. The radical of $B_{\lambda}$, i.e., the set of vectors $x$ such that $B_{\lambda}(x, y)=0$ for all $y \in \mathfrak{N}$ is the Lie algebra of the isotropy group of $\lambda$. A subspace $X \subseteq \mathfrak{R}$ is isotropic for $B_{\lambda}$ if $B_{\lambda_{\mid X}}$ is identically zero. The maximal isotropic subspaces for $B_{\lambda}$ are just the isotropic subspaces of maximum possible dimension, which is $1 / 2(\operatorname{dim} \mathfrak{R}+\operatorname{dim} \Re)$, where $\Re$ is the radical of $B_{\lambda}$. By a polarization of $\lambda$ we mean a subspace $\mathfrak{M}$ of $\mathfrak{R}$ which is maximal isotropic for $B_{\lambda}$ and which is a subalgebra of $\mathfrak{N}$. If $\mathfrak{M}$ is a polarization of $\lambda$, define $\psi=\psi_{\lambda}$ on $M$ by $\psi(\exp m)=$ $e^{2 \pi i \lambda(m)}$ for $m \in \mathfrak{M}$. Then $\psi$ is an unitary character of $M$. The representation $\rho=\rho(\mathcal{O})$ corresponding to $\mathcal{O}$ may be realized as the induced representation $\operatorname{ind}_{M}^{N} \psi$.

We will refer to the triple $(N, M, \psi)$ as a polarizing triple for $\rho$. We might also refer to $(\mathfrak{N}, \mathfrak{M}, \lambda)$ as a polarizing triple for $\rho$. Since $\psi$ does not determine $\lambda$, but only $\lambda_{\mid m}$, the choice of $\lambda$ in $(\mathfrak{N}, \mathfrak{M}, \lambda)$ might seem ambiguous. However, it can be shown [14] that if $\lambda_{\mid m}^{\prime}=\lambda_{\mid \Re}$, then $\lambda^{\prime}$ is in the $\mathrm{Ad}^{*} M$ orbit of $\lambda$, so the ambiguity in $\lambda$ is unimportant and we will ignore it. Alternatively, we might consider only $\lambda_{\mid \mathfrak{m}}$, and just regard $\lambda$ as an element of $\mathfrak{M}^{*}$.

If $\mathcal{O}$ is an $\mathrm{Ad}^{*} N$ orbit in $\mathfrak{N}^{*}$, then the linear span $Y$ of $\mathcal{O}$ is $\mathrm{Ad}^{*} N$-invariant. So, therefore, $Y^{\perp}=\Re$, the annihilator of $Y$ in $\Re$, is an ideal. Clearly $K=\exp \Omega$ is the identity component of the kernel of $\rho(\mathcal{O})$. Hence $\rho$ factors to $N / K$, and is locally faithful there, that is, has discrete kernel. In particular, if $\rho(\mathcal{O})$ is locally 
faithful, then $\mathcal{O}$ spans $\mathfrak{R}^{*}$. This is the same as to say that $\lambda \in \mathcal{O}$ is cyclic for $\mathrm{Ad}^{*} N$.

Let $M \cong N$ be a normal subgroup. Then $\mathcal{O}_{\mid m}=\left\{\mu_{\mid m}: \mu \in \mathcal{O}\right\}$, the projection of $\mathcal{O}$ on $\mathfrak{M}^{*}$, is a union of $\mathrm{Ad}^{*} M$ orbits, and the representations of $M$ corresponding to these orbits are the components into which $\rho(\mathcal{O})_{\mid M}$ decomposes. Again $\rho(\mathcal{O})_{\mid M}$ is locally faithful if and only if $\mathcal{O}_{\mathfrak{m}}$ spans $\mathfrak{M}^{*}$. If $\lambda \in \mathcal{O}$, then the orthogonal complement of $\mathfrak{M}$ with respect to $B_{\lambda}$ is the Lie algebra of the isotropy group of $\lambda_{\mid m}$.

Let $\mathscr{Z}(\mathfrak{R})^{\perp} \cong \mathfrak{N}^{*}$ be the annihilator of $\mathscr{Z}(\mathfrak{R})$. Given $\lambda \in \mathfrak{N}^{*}$, the $\operatorname{Ad}^{*} N$ orbit $\mathscr{O}$ through $\lambda$ will be contained in $\lambda+\mathscr{L}(\mathfrak{R})^{\perp}$. Thus in order for $\mathcal{O}$ to span $\mathfrak{R}^{*}$ it is necessary (and sufficient) that $\operatorname{dim} \mathscr{Z}(\mathfrak{R})=1$, and that $\lambda \notin \mathscr{Z}(\mathfrak{N})^{\perp}$. If this is so, then any normal $M \subseteq N$ will necessarily contain $\mathscr{2}(N)$.

The possibility that $\rho(\mathcal{O})$ be square integrable (see $\S 3$ ) is of particular interest to us. According to the criterion of Moore and Wolfe [11], $\rho$ will be square-integrable modulo $\mathscr{z}(N)$ precisely when $\mathscr{O}=\lambda+\mathscr{L}(\mathfrak{N})^{\perp}$. In that case, any irreducible representation defining on $\mathscr{Z}(N)$ the same unitary character as $\rho$ is equal to $\rho$. This is a generalization of the Stone-von Neumann theorem. We will refer to $\rho$ as having the Stone-von Neumann property.

We will be dealing in this section mainly with relations between nilpotent groups and associative nilpotent algebras. For these, we will need some notation parallel to that for groups. If $S_{0}$ is a nilpotent associative algebra, then $S=\boldsymbol{R} \oplus S_{0}$ will be the algebra obtained from $S_{0}$ by adjunction of the identity. Then $S_{0}$ forms an ideal in $S$, the Jacobson radical of $S$, the set of noninvertible elements. $S_{0}^{i}$ will be the ideal spanned by all products of $i$ or more elements from $S_{0}$. It is easy to see that a subspace $X \subseteq S_{0}$ generates $S_{0}$ as algebra if and only if $X+S_{0}^{2}=S_{0}$. The ideal $\left\{s \in S_{0}: s t=0\right.$, all $\left.t \in S_{0}\right\}$ will be denoted $\eta\left(S_{0}\right)$. We call it the null-ideal of $S_{0}$.

We begin our development with a very easy general remark concerning linear actions of nilpotent groups.

Proposition 2.1. (a) Let beV a real vector space. Let $A \subseteq G L(V)$ be a group of unipotent transformations. Let $S=S(A)$ be the linear span of $A$ in End $(V)$. Then

(i) $S$ is an associative subalgebra of End $(V)$.

(ii) $S=\boldsymbol{R} \oplus S_{0}$, where $\boldsymbol{R}$ here denotes the scalar matrices, and $S_{0}$ is the Jacobson radical of $S$, the ideal of nilpotent of noninvertible elements. $S_{0}$ is a nilpotent algebra.

(iii) The Lie algebra $\mathfrak{A}$ of $A$ is a Lie subalgebra of $S_{\imath}$. Moreover, $S_{0}=\mathfrak{A}+S_{0}^{2}$. Also $A \leqq 1+S_{0}$.

(b) Suppose that the action of $A$ is cyclic, that is, there is a 
vector $v \in V$ such that $A(v)$ spans $V$. Then

(iv) The map $e_{v}: S \rightarrow V$ given by $e_{v}(s)=s(v)$ is surjective. The kernel of $e_{v}$ is the left ideal $K_{v}=\{k \in S: k(v)=0\}$. Thus $V$ is isomorphic (via $e_{v}$ ) to $S / K_{v}$ as an $S$-module.

(v) The isotropy group of $v$ in $A$ has Lie algebra $\mathfrak{A} \cap K_{v}$.

(vi) $K_{v}$ contains no two-sided ideals in $S_{0}$.

(c) If $A$ is abelian, so is $S$. If $A$ acts cyclically with cyclic vector $v$, then $K_{v}=\{0\}$ so that $V \cong S$ as S-module. In particular, the isotropy group of $v$ in $A$ is trivial. Furthermore, $S$ is a maximal abelian nilpotent subalgebra of End $(V)$. There is thus a 1-1 correspondence between conjugacy classes of maximal abelian unipotent cyclic subgroups of $G L_{n}(\boldsymbol{R})$ and isomorphism classes of nilpotent commutative algebras of dimension $n-1$.

If $N$ is a nilpotent Lie group, and $M$ a subgroup, then we will refer to the pair $(N, M)$ simply as an $n$-pair. We will modify this term with adjectives modifying $M$. For example, if $M$ is normal, we have a normal $n$-pair; if $M$ is also abelian, then we have a normal abelian n-pair; and so on. We may substitute Lie algebras for groups in these definitions.

Suppose $(N, M)$ is a maximal normal abelian $n$-pair. Then it is not hard to see that $M$ is its own centralizer in $N$. Therefore $N / M$ acts faithfully on $M$ via Ad. Therefore $\mathfrak{N}$ fits in an exact sequence

$$
0 \longrightarrow \mathfrak{M} \longrightarrow \mathfrak{N} \longrightarrow \mathfrak{A} \longrightarrow 1
$$

were $\mathfrak{A} \subseteq$ End $(\mathfrak{M})$ is a Lie subalgebra of nilpotent endomorphisms. Let $\alpha=\alpha(\mathfrak{R})$ be the cohomology class in $H^{2}(\mathfrak{U} ; \mathfrak{M})$ defining $\mathfrak{N}$. Let $S_{0}=S_{0}(\mathfrak{H})$ be the associative algebra generated by $\mathfrak{A}$. We call the data $\left(\mathfrak{M}, S_{0}, \mathfrak{A}, \alpha\right)$ the associative correlative of the maximal normal abelian $n$-pair $(N, M)$.

Proposition 2.2. Attaching the associative correlative (M, $\left.S_{0}, \mathfrak{A}, \alpha\right)$ to the maximal normal abelian n-pair $(N, M)$ establishes a bijection between such n-pairs and 4-tuples (MN, $\left.S_{0}, \mathfrak{A}, \alpha\right)$ such that

(i) $\mathfrak{M}$ is a real vector space.

(ii) $S_{0}$ is a subalgebra of End (M), consisting of nilpotent endomorphisms.

(iii) $\mathfrak{A}$ is a Lie subalgebra of $S_{0}$, such that $S_{0}=\mathfrak{A}+S_{0}^{2}$.

(iv) $\alpha$ is an element of $H^{2}(\mathfrak{A} ; \mathfrak{M})$.

Given these data, $\mathfrak{N}$ is defined by the sequence

$$
0 \longrightarrow \mathfrak{M} \longrightarrow \mathfrak{R} \longrightarrow \mathfrak{A} \longrightarrow 0
$$

defined by the class $\alpha$ and $\mathfrak{M}$ is the ideal in the maximal normal 
abelian n-pair ( $\mathfrak{N}, \mathfrak{M})$.

If $(N, M, \psi)$ is a polarizing triple for some representation $\rho$ of $N$, then we may attach any adjectives applying to the $n$-pair $(N, M)$ to the triple also. Additionally, if $\rho$ is locally faithful, we will call the triple faithful.

Proposition 2.3. (a) Suppose $(N, M, \psi)$ is a faithful normal polarization. Then $(N, M)$ is a maximal normal abelian pair. Let $\left(\mathfrak{M}, S_{0}, \mathfrak{A}, \alpha\right)$ be the associative correlative of $(N, M)$. Let $A=\exp \mathfrak{A}$. Let $\lambda \in \mathfrak{M}^{*}$ correspond to $\psi$. Then $A$, acting contragrediently on $\mathfrak{M}^{*}$, acts cyclically with cyclic vector $\lambda$. Moreover, the isotropy group of $\lambda$ in $A$ is trivial. Thus if $K_{\lambda}=\left\{s \in S_{0}, s^{*}(\lambda)=0\right\}$ where $s^{*}$ is the contragredient of $s$, we have $\mathfrak{M} \cong K_{\lambda}^{\perp} \subseteq S^{*}$, and $\mathfrak{A} \cap K_{\lambda}=\{0\}$.

(b) Suppose $\rho=\operatorname{ind}_{M}^{N} \psi$ is square-integrable modulo $\check{Z}(N)$. Then $\operatorname{dim} N=2 \operatorname{dim} M-1$, and $S_{0}=\mathfrak{A} \oplus K_{\lambda}$. In particular, if $N / M$ is abelian, $\mathfrak{A}=S_{0}$.

(c) If $N$ is metabelian, then any locally faithful representation of $N$ (these exist if and only if $\operatorname{dim} \not{z}=1$ ) allows a faithful normal polarization $(N, M, \psi)$ with the additional property that $N / M$ is abelian.

Thus 2.3 shows that a normal polarizing triple $(N, M, \psi)$ is built in a certain way from a nilpotent associative algebra $S_{0}$. The next propositions investigate the extent to which $S_{0}$ depends on $N$ alone, and how it varies with $M$. A companion question is of course how $M$ may vary, or somewhat more generally, what are all the possible polarizing triples for the $\rho$ defined by $(N, M, \psi)$.

Proposition 2.4. (a) Let $(\mathfrak{N}, \mathfrak{M}, \lambda)$ be a faithful normal polarizing triple. Let ( $\mathfrak{M}, S_{0}, \mathfrak{A}, \alpha$ ) be the associative correlative of $(\mathfrak{R}, \mathfrak{M})$. According to our discussion in the definition of polarizing triples, we consider that $\lambda \in \mathfrak{R}^{*}$. Let $\mathfrak{P}$ be any other polarization of $\lambda$. We may identify $\mathfrak{P} /(\mathfrak{P} \cap \mathfrak{M})$ to a subspace $\mathfrak{B}$ of $\mathfrak{A}$. The following facts hold.

(i) Under the isomorphism $\mathfrak{M} \cong K_{\lambda}^{\perp} \cong S^{*}$, the intersection $\mathfrak{P} \cap$ $\mathfrak{M}$ is identified to $K_{\lambda}^{\perp} \cap \mathfrak{B}^{\perp}=\left(K_{\lambda} \oplus \mathfrak{B}\right)^{\perp}$. (Here $\lambda$ signifies $\lambda_{\mid \mathfrak{m}}$.)

(ii) $\mathfrak{B}$ is a Lie subalgebra of $\mathfrak{A}$, and the associative algebra generated by $\mathfrak{B}$ is contained in $K_{\lambda} \oplus \mathfrak{B}$.

(iii) If $\mathfrak{P}$ is abelian, then $\mathfrak{B}$ is an abelian Lie subalgebra of $\mathfrak{A}$, and $\mathfrak{B} S \subseteq K_{\lambda} \oplus \mathfrak{B}$.

(iv) If $\mathfrak{F}$ is an ideal in $\mathfrak{R}$, then $\mathfrak{B}$ is an ideal in $\mathfrak{A}$, and $\mathfrak{B}\left(K_{\lambda} \oplus \mathfrak{B}\right) \subseteq K_{\lambda}$ and $K_{\lambda} \oplus \mathfrak{B}$ is a left ideal in $S$. Since from 2.3 we know $\mathfrak{P}$ is also abelian, we have also $S \mathfrak{B S} \subseteq K_{\lambda} \oplus \mathfrak{B}$. 
(b) If $\Re / \mathfrak{M}$, hence $S$, is abelian, these facts sharpen and simplify as follows.

(i ) Since $K_{\lambda}=\{0\}$, $\mathfrak{M} \cong S^{*}$ and $\mathfrak{P} \cap \mathfrak{M}=\mathfrak{B}^{\perp}$.

(ii) $\mathfrak{B}$ is an associative subalgebra of $S$.

(iii) If $\mathfrak{P}$ is abelian, then $\mathfrak{B}$ is an ideal in $S_{0}$, and is abelian as associative algebra.

(iv) If $\mathfrak{P}$ is an ideal in $\mathfrak{R}$, then $\mathfrak{B}^{2}=0$.

( v) If $\mathfrak{P}$ is an ideal and $\mathfrak{R} / \mathfrak{P}$ is also abelian, then $\mathfrak{B} S_{0}=0$.

The facts recited above indicate that the existence of groups other than $M$ from which to induce $\rho$ requires compatibility $\mathfrak{N}$ with the associative structure of $S$. This is clearest in the extreme case.

Proposition 2.5. With the notations of 2.4, suppose $\mathfrak{B}=\mathfrak{A}$, or in other words $\mathfrak{R}=\mathfrak{P}+\mathfrak{M}$. Then

(i) $\mathfrak{A}$ is a complement to $K_{\lambda}$ in $S_{0}$, i.e., $S_{0}=\mathfrak{A} \oplus K_{\lambda}$.

(ii) $\mathfrak{Y} \cap \mathfrak{M}=\mathscr{Z}(\mathfrak{R})$ (which is one-dimensional).

(iii) $\mathfrak{R}$ is isomorphic to the semidirect product $\mathfrak{A} \mathrm{X}_{s} K_{\lambda}^{\perp}$. (When $\mathfrak{N} / \mathfrak{M}$ is abelian, this is $S_{0} \times{ }_{s} S^{*}$.)

(iv) The representation $\rho=\operatorname{ind}_{M}^{N}$ \& corresponding to the triple $(\mathfrak{N}, \mathfrak{M}, \lambda)$ is square-integrable.

As we will see later on, it is when transversal polarizations exist for $\rho$ that the Fourier transform has a nice interpretation.

Finally we have in case $N / M$ is abelian a fairly definitive statement concerning how much of $S_{0}$ is determined by $N$ alone.

Proposition 2.6. (a) If $N$ is nilpotent metabelian, then $x \in N$ belongs to a normal abelian subgroup with abelian quotient if and only if $x$ centralizes $N^{(2)}$. The centralizer $C\left(N^{(2)}\right)$ of $N^{(2)}$ is a twostep nilpotent group containing $N^{(2)}$. A subgroup $M \subseteq C\left(N^{(2)}\right)$ is maximal normal abelian in $N$ with abelian quotient if and only if $M$ is maximal abelian in $C\left(N^{(2)}\right)$.

(b) Suppose $(N, M, \psi)$ is a faithful normal polarizing triple with abelian quotient. Let (M, $\left.S_{0}, \mathfrak{A}, \alpha\right)$ be the associative correlative of $(N, M)$. Then the image of $C\left(\mathfrak{N}^{(2)}\right) / \mathfrak{M}$ in $\mathfrak{A}$ is contained in the null-ideal $\eta\left(S_{0}\right)$. Thus the image of $S_{0}$ in Hom $\left(\mathfrak{R}^{(2)}\right)$, which is the algebra generated by Ad $N_{\mid \Re(2)}$ and which is canonically attached to $N$ (i.e., does not depend on $M$ ) is isomorphic to $S_{0} / I$ where $I$ is a subspace of $\eta\left(S_{0}\right)$.

(c) Suppose the representation attached to $(N, M, \psi)$ is squareintegrable modulo $\mathscr{Z}(N)$. Let $Y \subseteq \mathfrak{N}$ be any linear complement to $C\left(\mathfrak{N}^{(2)}\right)$. Put $\mathfrak{N}_{1}=Y \oplus \mathfrak{N}^{(2)}$. Then $N_{1}$ is normal in $N$, and $\left(N_{1}, N^{(2)}, \psi_{\mid N^{(2)}}\right)$ is a faithful normal polarizing triple yielding a 
square-integrable representation of $N_{1}$. Choose $\lambda \in \Re_{1}^{*}$, such that $\lambda_{\mid \Re(2)}$ corresponds to $\psi_{\mid N}(2)$. Let $H$ be the isotropy group of $\lambda$ in $\mathrm{Ad}^{*} N$ acting on $\mathfrak{N}_{1}^{*}$. Then $H$ is an Heisenberg group, and we have the relations $N=H \cdot N_{1}$, and $H \cap N_{1}=\mathscr{Z}(N)=\mathscr{Z}\left(N_{1}\right)=\mathscr{Z}(H)$.

We now specialize to a particular class of groups which seem particularly relevant to the harmonic analysis we wish to do. We will call a nilpotent group $N$ an HAT group if

(i) $N$ is metabelian.

(ii) $N$ has a locally faithful representation which is squareintegrable modulo $\mathscr{z}$.

Let $N$ be an HAT group, and fix a representation $\rho$ of $N$ satisfying (ii) above. Let $(N, M, \psi)$ be a polarizing triple corresponding to $\rho$. According to Proposition 2.3(c), we may assume $M \supseteqq N^{(2)}$, so $(N, M)$ is a normal $n$-pair with abelian quotient. Let (M, $\left.S_{0}, \mathfrak{A}, \alpha\right)$ be the associative correlative to $(N, M)$. Then from Proposition 2.3(b), and 2.4(b) we see that $\mathfrak{A}=S_{0}$ and $\mathfrak{M} \cong S^{*}$ so that $\mathfrak{R}$ fits into an exact sequence $0 \rightarrow S^{*} \rightarrow \mathfrak{N} \rightarrow S_{0} \rightarrow 0$, such that the action of $S_{0}$ on $S^{*}$ is the contragredient of left multiplication on $S$. Moreover, if $\lambda$ is the linear functional on $\mathfrak{M}$ corresponding to $\psi$, then the identification of $\mathfrak{M}$ with $S^{*}$ may be accomplished (i.e., normalized) in such a way that $\lambda$ is identified to $1 \in S$.

Continuing, we see from Proposition 2.6(b) and (c) that although $S_{0}$ is not uniquely determined by $N$, a certain quotient $S_{0} / I$, with $I \subseteq \eta\left(S_{0}\right)$, is determined by $N$. Let $N_{1} \subseteq N$ be a normal subgroup constructed as in 2.6(c). Then $N_{1}$ is also an HAT group, possessing the normal faithful polarizing triple $\left(N_{1}, N^{(2)}, \psi_{\mid N^{(2)}}\right)$. The associative correlative of the $n$-pair $\left(N_{1}, N^{(2)}\right)$ is $\left(\mathfrak{R}^{(2)}, S_{0} / I, S_{0} / I, \alpha^{\prime}\right)$ where $\alpha^{\prime}$ is whatever it must be.

Again with an eye towards harmonic analysis, we are interested in computing the automorphisms of the HAT group $N$. If $N_{1}$ is as in the preceding paragraph, then $C\left(N^{(2)}\right) \subseteq N$ provides, via $\mathrm{Ad}$, some outer automorphisms of $N_{1}$ which are trivial on $N^{(2)}$ and on $N_{1} / N^{(2)}$. We will see below that automorphisms of this sort always result from embeddings of one HAT group as normal subgroup of another.

For the automorphism computations, we will offer proofs. From Proposition 2.6(b) we see that one question of interest for the structure of HAT groups is to describe for a given commutative nilpotent ássociative algebra $S_{0}$ the possible nilpotent algebras $T_{0}$ such that $S_{0} \cong T_{0} / J$, with $J \cong \eta\left(T_{0}\right)$. This point is also important for the description of automorphisms, and is covered by the next proposition. 
Proposition 2.7. (a) For any finite-dimensional nilpotent associative commutative algebra $S_{0}$, there is another such algebra $\widetilde{S}_{0}$ and an exact sequence of algebras

$$
0 \longrightarrow I \longrightarrow \widetilde{S}_{0} \stackrel{q}{\longrightarrow} S_{0} \longrightarrow 0
$$

with $I \subseteq \eta\left(\widetilde{S}_{0}\right) \cap \widetilde{S}_{0}^{2}$ and possessing the following universal property. For any exact sequence of algebras

$$
0 \longrightarrow J \longrightarrow T_{0} \stackrel{a}{\longrightarrow} S_{0} \longrightarrow 0
$$

(with $T_{0}$ finite-dimensional, nilpotent, commutative and associative), and $J \subseteq \eta\left(T_{0}\right) \cap T_{0}^{2}$, there is a surjective homomorphism $\widetilde{a}: \widetilde{S}_{0} \rightarrow T_{0}$ such that

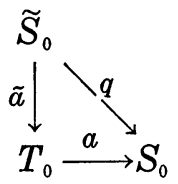

commutes. In particular, $\widetilde{S}_{0}$ in unique up to isomorphism.

(b) If $0 \rightarrow J \rightarrow T_{0} \rightarrow S_{0} \rightarrow 0$ is any exact sequence of commutative nilpotent algebras, with $J \subseteq \eta\left(T_{0}\right)$, then we can write $T_{0}=$ $T_{0}^{\prime} \oplus X$, a direct sum of algebras such that $J=X \oplus J \cap T_{0}^{2}$, and $T_{0}^{\prime} / T_{0}^{\prime 2} \oplus X=T_{0} / T_{0}^{2}$, or in other terms, $T_{0}^{2}=T_{0}^{\prime 2}$.

Proof. (a) Let $U$ be any complement to $S_{0}^{2}$ in $S_{0}$. Let $S(U)=$ $\mathscr{S}$ be the symmetric algebra over $U$, and let $\mathscr{S}_{0}$ be the ideal in $\mathscr{S}$ generated by $U$. Then inclusion $U \subseteq S_{0}$ induces a surjective homomorphism $\sigma: \mathscr{S}_{0} \rightarrow S_{0}$. The kernel of $\sigma$ will be some ideal $\mathscr{I} \subseteq \mathscr{S}_{0}$, so that $S_{0} \cong \mathscr{S}_{0} / \mathscr{F}_{\text {. }}$ Put $\tilde{\mathscr{I}}=U \mathscr{F}=\mathscr{S}_{0} \mathscr{F}$, and put $\tilde{S}_{0}=\mathscr{S}_{0} / \tilde{\mathscr{F}}$. Then if $I \cong \mathscr{I} / \tilde{\mathscr{F}}$, we have the exact sequence

$$
0 \longrightarrow I \longrightarrow \widetilde{S}_{0} \stackrel{q}{\longrightarrow} S_{0} \longrightarrow 0
$$

and $I \subseteq \eta\left(\widetilde{S_{0}}\right) \cap S_{0}^{2}$. The claim is that this $\widetilde{S_{0}}$ is the $\widetilde{S_{0}}$ we want.

Let $0 \rightarrow J \rightarrow T_{0} \stackrel{a}{\rightarrow} S_{0} \rightarrow 0$ be an exact sequence of the sort we are discussing. Choose a space $U^{\prime} \cong T_{0}$ such that $\alpha: U^{\prime} \rightarrow U$ is an isomorphism of vector spaces. Our assumption on $J$ shows that $U^{\prime}$ exists. The inverse map $a^{-1}: U \rightarrow U^{\prime} \subseteq T_{0}$ induces a homomorphism $\tau: \mathscr{S}_{0} \rightarrow T_{0}$. Our assumptions imply $\tau$ is surjective. Moreover, by construction the triangle

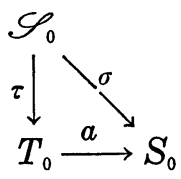


commutes. Therefore $\tau(\mathscr{J}) \subseteq J$. Since $J \subseteq \eta\left(T_{0}\right)$, we have $\tilde{\mathcal{J}} \subseteq$ $\operatorname{ker} \tau$. Thus $\tau$ factors to a map $\tilde{a}: \widetilde{S}_{0} \rightarrow T_{0}$ as desired.

For part (b), consider again the exact sequence

$$
0 \longrightarrow J \longrightarrow T_{0} \longrightarrow S_{0} \longrightarrow 0
$$

in which we now assume only that $J \subseteq \eta\left(T_{0}\right)$, not that $J \subseteq T_{0}^{2}$. Let $X$ be a complement to $J \cap T_{0}^{2}$ in $J$, so that $J=\left(J \cap T_{0}^{2}\right) \oplus X$. Let $U$ be a complement to $T_{0}^{2} \oplus X$ in $T_{0}$. Then $U \oplus X$ is a complement to $T_{0}^{2}$ in $T_{0}$, so $U \oplus X$ generates $T_{0}$. Thus if $T_{0}^{\prime}$ is the subalgebra generated by $U$, then $T_{0}=T_{0}^{\prime} \oplus X$, since $X \subseteq \eta\left(T_{0}\right)$. This finishes the proposition.

Now turn to direct consideration of automorphisms of an HAT group $N$. Let $(N, M, \psi)$ be a normal polarizing triple with abelian quotient for a locally faithful square-integrable representation $\rho$ of $N$. Let $\lambda \in \mathfrak{N}^{*}$ be in the orbit corresponding to $\rho$. Then as vector space, $\mathfrak{N}$ has the direct sum decomposition $\mathfrak{N}=\mathscr{Z}(\mathfrak{R}) \oplus \operatorname{ker} \lambda$. If $Y$ is any other complement to $\mathscr{Z}(\mathfrak{R})$ in $\mathfrak{N}$, then $Y=\operatorname{ker} \lambda^{\prime}$ for some $\lambda^{\prime} \in \lambda+\mathscr{Z}(\mathfrak{R})^{\perp}$. But $\lambda+\mathscr{Z}(\mathfrak{R})^{\perp}=\operatorname{Ad}^{*} N(\lambda)$ since $\rho$ is square-integrable, so Ad $N$ acts simply transitively on linear complements to $\mathscr{Z}(\mathfrak{N})$ in $\mathfrak{N}$. Thus if Aut $(N, \lambda)$ is the subgroup of Aut $N$ which preserves $\operatorname{ker} \lambda$, we have $\operatorname{Aut}(N) \cong \operatorname{Aut}(N, \lambda) \times_{s} \operatorname{Ad} N$. Thus we will describe $\operatorname{Aut}(N, \lambda)$. We note that $\operatorname{Aut}(N, \lambda)$ is a subgroup of the "symplectic similitudes" of the form $B_{\lambda}$ on ker $\lambda$.

Let $\left(S^{*}, S_{0}, S_{0}, \alpha\right)$ be the associative correlative to the $n$-pair $(N, M)$. Then as we saw above, $N$ fits in the exact sequence

$$
1 \longrightarrow S^{*} \longrightarrow N \longrightarrow 1+S_{0} \longrightarrow 1 \text {. }
$$

Let Aut $S$ be the group of algebra automorphisms of $S$, and let $\operatorname{Aut}(N, M, \lambda)$ be the subgroup of $\operatorname{Aut}(N, \lambda)$ also preserving $M$. Let $r:$ Aut $(N, M, \lambda) \rightarrow G L(M)$ be the restriction map.

Proposition 2.8. (a) When $M$ is identified to $S^{*}$, the group $r$ (Aut $(N, M, \lambda)$ ) is identified to a subgroup of $\boldsymbol{R}^{x} \times \operatorname{Aut}(S)^{*}$, where $\boldsymbol{R}^{x}$ is the scalars, and Aut $(S)^{*}$ is Aut $S$ acting contragrediently on $S^{*}$. Moreover, if $r$ (Aut $(N, M, \lambda)$ ) contains a scalar operator, then $N$ is the twisted direct product $\left(1+S_{0}\right) \times_{s} S^{*}$. In this case, $r($ Aut $(N, M, \lambda))$ is all of $\boldsymbol{R}^{x} \times$ Aut $S$. Otherwise, it is isomorphic to a subgroup of Aut $S$.

(b) The kernel of $r$ acts trivially on $M$ and on $N / M$, and is isomorphic to $H^{1}\left(S, S^{*}\right)$ (Lie algebra cohomology). In turn, $H^{1}\left(S, S^{*}\right)$ is isomorphic to $I^{*}$, where $I=I\left(S_{0}\right)$ is the ideal of Proposition 2.7(a).

Proof. It will be more convenient to work with $\mathfrak{N}$ than with 
$N$. We make the obvious alterations in notation. Choose $\alpha \in$ Aut $(\mathfrak{N}, \mathfrak{M}, \lambda)$. Let $\alpha_{1}=r(\alpha)$ and let $\alpha_{2}$ be the factorization of $\alpha$ to $\mathfrak{N} / \mathfrak{M}$. We identify $\mathfrak{M}$ and $\mathfrak{R} / \mathfrak{M}$ with $S^{*}$ and $S_{0}$ respectively and so regard the $\alpha_{i}$ as transformations on these spaces. As we have remarked, we may assume $\lambda_{\mid m}$ is identified to $1 \in S$. Then if $\alpha_{1}^{*} \in$ End $(S)$ is the contragredient of $\alpha_{1}$, we will have $\alpha_{1}^{*}(1)=t=t \cdot 1$ for some $t \in \boldsymbol{R}$, since $\lambda$ is an eigenvector for $\operatorname{Aut}(N, M, \lambda)$ by definition. If $n \in \mathfrak{N}$ and $m \in \mathfrak{M}$, then $[n, m]$ depends only on $n$ modulo $\mathfrak{M}$. If $n$ corresponds to $s \in S_{0}$ and $m$ to $\nu \in S^{*}$, then $[n, m]$ corresponds to $m^{*}(s)(\nu)$, where $m^{*}(s)(\nu)\left(s^{\prime}\right)=\nu\left(-s s^{\prime}\right)$. Thus we have $\alpha_{1}\left(m^{*}(s)(\nu)\right)=m^{*}\left(\alpha_{2}(s)\right) \alpha_{1}(\nu)$. In other words

$$
\alpha_{1}\left(m^{*}(s)(\boldsymbol{\nu})\right)\left(s^{\prime}\right)=m^{*}(s)(\boldsymbol{\nu})\left(\alpha_{1}^{*-1}\left(s^{\prime}\right)\right)=\boldsymbol{\nu}\left(-s \alpha_{1}^{*-1}\left(s^{\prime}\right)\right)
$$

and this equals

$$
m^{*}\left(\alpha_{2}(s)\right) \alpha_{1}(\nu)\left(s^{\prime}\right)=\nu\left(\alpha_{1}^{*-1}\left(-\alpha_{2}(s) s^{\prime}\right)\right) .
$$

Since $\nu$ is arbitrary, this implies

$$
-s \alpha_{1}^{*-1}\left(s^{\prime}\right)=\alpha_{1}^{*-1}\left(-\alpha_{2}(s) s^{\prime}\right) \text { for } s \in S_{0} \text { and } s^{\prime} \in S .
$$

Write $s^{\prime}=\alpha_{1}^{*}\left(s^{\prime \prime}\right)$, and transform both sides by $\alpha_{1}^{*}$ to obtain

$$
\alpha_{1}^{*}\left(s s^{\prime \prime}\right)=\alpha_{2}(s) \alpha_{1}^{*}\left(s^{\prime \prime}\right) \text { for } s \in S_{0} \text { and } s^{\prime \prime} \in S .
$$

In this identity, put $s^{\prime \prime}=1$ to conclude

$$
\alpha_{1}^{*}(s)=t \alpha_{2}(s) \text { for } s \in S_{0} .
$$

Putting this back into (1) we find that $t^{-1} \alpha_{1}^{*}$ is an automorphism of $S$, establishing the first statements in each of parts (a) and (b) of the proposition.

Next, suppose $\alpha_{1}(\nu)=t \nu$ for all $\nu \in S^{*}$. Then $\alpha_{2}(s)=s$ and we may write $\mathfrak{N}=S_{0} \oplus S^{*}$ where $S_{0}$ is the 1-eigenspace of $\alpha$ and $S^{*}$ is the $t$-eigenspace of $\alpha$. Since $\alpha$ is an automorphism, $S_{0}$ is a subalgebra of $\mathfrak{N}$, and therefore we have exhibited $\mathfrak{N}$ as the twisted direct product of $S_{0}$ and $S^{*}$. Conversely, when $\mathfrak{N}$ is split, the computations above read backwards guarantee that $r(\operatorname{Aut}(N, M, \lambda))$ is indeed all of $\boldsymbol{R}^{x} \times \operatorname{Aut}(S)^{*}$. This concludes the proof of (a).

To identify $\operatorname{ker} r_{B}$, let us recall an interpretation of $H^{1}$ which is perhaps less in the public consciousness than some. Let $\mathfrak{A}$ be a Lie algebra and let $X$ be an $\mathfrak{A}$-module. Denote the action of $\mathfrak{A}$ on $X$ by $\rho$. The space $C^{1}(\mathscr{X}: X)$ is defined as the set of linear maps $\tau: \mathfrak{A} \rightarrow X$ satisfying $\tau([a, b])=\rho(a) \tau(b)-\rho(b) \tau(a)$. The subspace $B^{1}(\mathfrak{A}, X)$ of $C^{1}$ consists of $\tau$ of the form $\tau(a)=\rho(a) x$ for some $x \in X$. The quotient $C^{1} / B^{1}$ is the cohomology group $H^{1}(\mathfrak{A} ; X)$.

Consider any Lie algebra $(\$)$ which fits into an exact sequence 


$$
0 \longrightarrow X \longrightarrow \mathfrak{B S} \longrightarrow \mathfrak{A} \longrightarrow 0
$$

such that the action of $A$ induced on $X$ by Ad is just $\rho$. We may write $\mathbb{B}=\mathfrak{A} \oplus X$ as vector spaces, and the bracket operation in $\mathbb{S}$, denoted by $[,]^{0}$ will be as follows:

$$
\begin{aligned}
& {[x, y]^{0}=0 \text { for } x, y \in X ;} \\
& {[a, x]^{0}=\rho(a) x \text { for } x \in X \text { and } a \in \mathfrak{R}: \text { and }} \\
& {[a, b]^{0}=[a, b]+\gamma(a, b) .}
\end{aligned}
$$

In the last expression, $[a, b]$ is the usual bracket in $\mathfrak{A}$, and $\gamma$ is an appropriate antisymmetric bilinear map from $\mathfrak{A}$ to $X$.

Suppose that $\sigma$ is an automorphism of $\&$ which is the identity on $X$ and induces the identity map on $\mathbb{S} / X$. Then we can write $\sigma(a)=a+\tau(a)$ for $a \in \mathfrak{A}$, where $\tau$ is a linear map from $\mathfrak{A}$ to $X$. In order that $\sigma$ be an automorphism we must have $\sigma\left([a, b]^{0}\right)=[\sigma(a), \sigma(b)]^{0}$. Expanding this identity out by means of the formulas above, we find the equation

$$
[a, b]+\gamma(a, b)+\tau([a, b])=[a, b]+\gamma(a, b)+\rho(a) \tau(b)-\rho(b) \tau(a) .
$$

Cancelling common terms, we find just the requirement that $\tau$ belong to $C^{1}(\mathscr{A} ; X)$. This space is thus canonically identified to automor-

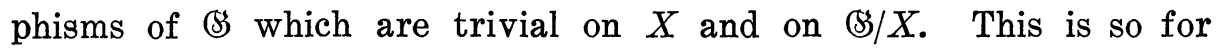
any possible 8 .

Among the automorphisms of (S) associated to $C_{1}(\mathfrak{H} ; X)$, some will be inner antomorphisms of the form exp ad $x, x \in X$. Indeed, if $x \in X$, then ad $x^{2}=0$ so exp ad $x=1+$ ad $x$. Thus exp ad $x(a)=$ $a+[x, a]=a-\rho(a) x$. Therefore $B^{1}(\mathfrak{A} ; X)$ is identified to the inner automorphisms attached to $C_{1}(\mathfrak{A} ; X)$. So finally, $H^{1}(\mathfrak{A} ; X)$ is just the group of outer automorphisms which are trivial on $X$ and on $\$ \$ Y$.

In the light of this general discussion, we see that $\operatorname{ker} r$, since it forms a complement to $\operatorname{Ad} M=B^{1}\left(S_{0} ; S^{*}\right)$ in $C^{1}\left(S_{0}, S^{*}\right)$, by virtue of the discussion preceding this proposition, is indeed isomorphic to $H^{1}\left(S_{0} ; S^{*}\right)$.

Therefore to complete the proposition, it remains only to compute $H^{1}\left(S_{0}, S^{*}\right)$. Since $S_{0}$ is abelian, the cocycle identity for $\tau \epsilon$ $C\left(S_{0}, S^{*}\right)$ reduces to $m^{*}(x) \tau(y)=m^{*}(y) \tau(x)$ for $x, y \in S_{0}$. We define a bilinear form $F_{\tau}$ on $S \times S_{0}$ by the rule $F_{\tau}(z, x)=\tau(x)(z)$. The cocycle identity then says

$$
F_{\tau}(z x, y)=F_{\tau}(z y, x) \text {. }
$$

In other words, the map $(x, y) \rightarrow F_{\tau}(z x, y)$ is a symmetric bilinear form on $S_{0} \times S_{0}$ for any $z \in S$. Let $\widetilde{S_{0}}$ be the universal null-extension of $S_{0}$ constructed in Proposition 2.7(a). We have the exact 
sequence

$$
0 \longrightarrow I \longrightarrow \widetilde{S}_{0} \stackrel{q}{\longrightarrow} S_{0} \longrightarrow 0
$$

where $I \leqq \eta\left(S_{0}\right)$. Choose a linear cross-section $\sigma$ to $q$. Then $\sigma(x) \sigma(y)=$ $\sigma(x y)+\delta(x, y)$, where $\delta(x, y) \in I$. The map $\delta: S_{0} \times S_{0} \rightarrow I$ is evidently bilinear and symmetric, and in fact, $\delta(z x, y)$ is symmetric in $x$ and $y$ for any $z \in S$. Thus if $\lambda \in I^{*}$, we see $\lambda \circ \delta$ is a bilinear form coming from a cocycle in $C^{1}\left(S_{0}, S^{*}\right)$. Thus we have a natural map $\beta: I^{*} \rightarrow H^{1}\left(S_{0}, S^{*}\right)$. I claim $\beta$ is an isomorphism. Indeed, if $F_{\tau}$ is the bilinear form of a cocycle, put $T_{0}=S_{0} \oplus L$, where $L$ is a line with basis element $l$. Define a multiplication $\circ$ on $T_{0}$ by the rules

$$
\begin{aligned}
& s \circ s^{\prime}=s s^{\prime}+F_{\tau}\left(s, s^{\prime}\right) l \text { for } s, s^{\prime} \in S_{0}, \text { and } \\
& l \circ l=s \circ l=0 .
\end{aligned}
$$

The properties of $F_{\tau}$ make $\circ$ into a commutative associative multiplication with $L \leqq \eta\left(T_{0}\right)$. Further straightforward computation shows $L \nsubseteq T_{0}^{2}$ if and only if $\tau$ is a coboundary, in which case $F_{\tau}(x, y)=$ $\mu(x y)$ for some $\mu \in S_{0}^{*}$. This completes Proposition 2.8.

As a consequence of our study of automorphisms, we obtain the following uniqueness result. We say a faithful normal polarizing triple with abelian quotient $(N, M, \psi)$ is split if in the associative correlative ( $\left.\mathfrak{M}, S_{0}, S_{0}, \alpha\right)$ to $(N, M)$, the extension class $\alpha$ is trivial, that is, $\mathfrak{R}$ is isomorphic to $S_{0} \cdot \boldsymbol{X}_{s} S^{*}$ as Lie algebra. If $N$ allows such a split triple, then we say $N$ is a split HAT group.

Proposition 2.9. (a) There is a bijection between isomorphism classes of split HAT groups and isomorphism classes of abelian nilpotent associative finite dimensional algebras. In other words, if $N$ is a split HAT group with split (normal faithful polarizing) triple $(N, M, \psi)$ (with abelian quotient), and if (M, $\left.S_{0}, S_{0}, 0\right)$ is the associative correlative, then $S_{0}$ is determined by $N$. That is, if $\left(N, M^{\prime}, \psi^{\prime}\right)$ is another split triple with associative correlative $\left(\mathfrak{M}^{\prime}, S_{0}^{\prime}, S_{0}^{\prime}, 0\right)$, then $S_{0}^{\prime}$ is isomorphic to $S_{0}$.

(b) In fact, if $\eta\left(S_{0}\right) \subset S_{0}^{2}$, then $\eta\left(S_{0}\right) \cap C\left(\mathscr{N}^{(2)}\right)$ is uniquely determined modulo $\mathscr{N}^{(2)}$. In particular, if $\left(\mathscr{L}^{\prime}, S_{0}^{\prime}, S_{0}^{\prime}, 0\right)$ is another associative correlative for $\mathscr{N}$, then $\mathscr{N}=S_{0}+\mathscr{C}^{\prime}=S_{0}^{\prime}+\mathscr{C}^{\prime}$, so dividing $\mathscr{N}$ by $\mathscr{C}^{\prime}$ defines an algebra isomorphism form $S_{0}$ to $S_{0}^{\prime}$,

Proof. Write $\mathscr{N}=S_{0} \oplus S^{*}$. It is clear that when $\mathscr{N}$ is split $\mathscr{N}^{(2)}=\eta\left(S_{0}\right)^{\perp}$ exactly. Consider $\mathscr{Z}^{(2)}(\mathscr{N})$. Since $\mathscr{Z}(\mathscr{N})$ is onedimensional, an element $n$ in $\mathscr{N}$ can be in $\mathscr{Z}^{(2)}(\mathscr{N})$ only if the image of ad $n$ is one-dimensional. Take $s \in S$. Then the kernel of ad $s$ acting on $S^{*}$ is $(s S)^{\perp}$. Thus $s$ can belong to $\mathscr{L}^{(2)}(\mathscr{N})$ only if 
$s \in \eta\left(S_{0}\right)$. Conversely, when $\mathscr{N}$ is split, then ad $s(\mathscr{N})=\operatorname{ad} s\left(S^{*}\right)=S_{0}^{\perp}$ for $s \in \eta\left(S_{0}\right)$. Thus

$$
S_{0} \cap \mathscr{Z}^{(2)}(\mathscr{N})=S_{0} \cap C\left(\mathscr{N}^{(2)}\right)=\eta\left(S_{0}\right)
$$

(If $\mathscr{N}$ is not split, these equalities are inclusions.) A general element of $\mathscr{Z}^{(2)}(\mathscr{N})$ has the form $s+\lambda$ with $s \in S_{0}$ and $\lambda \in S^{*}$. Since

$$
\operatorname{ad}(s+\lambda)(\mathscr{N}) \supseteqq \operatorname{ad}(s+\lambda)\left(S^{*}\right)=\operatorname{ad} s\left(S^{*}\right)
$$

we see already that $s \in \mathscr{Z}^{(2)}(\mathscr{N})$ by our analysis above. Hence

$$
\mathscr{Z}^{(2)}(\mathscr{N})=\eta\left(S_{0}\right) \oplus\left(\left(\mathscr{\digamma}^{(2)}(\mathscr{N}) \cap S^{*}\right)\right. \text {. }
$$

For $\lambda \in S^{*}$, we have in ad $\lambda=\left\{\lambda \circ s: s \in S_{0}\right\}$. Evidently $\lambda \circ s$ belongs to $\mathscr{Z}(\mathscr{N})=S_{0}^{\perp}$ for all $s$ if and only if $\lambda\left(s_{1} s_{2}\right)=0$ for all $s_{1}$ and $s_{2}$ in $S_{0}$. Thus

$$
\mathscr{\check { Z }}^{(2)}(\mathscr{N}) \cap S^{*}=\left(S_{0}^{2}\right)^{\perp}
$$

Consider for a moment a general Lie algebra $\mathscr{N}$ with onedimensional center. The bracket on $\mathscr{Z}^{(2)}(\mathscr{N})$ is then an alternating $\mathscr{F}(\mathscr{N})$-valued form, the radical of which is the center $\mathscr{F}^{(2)}(\mathscr{N})$. If $Y$ is any subspace of $\mathscr{Z}^{(2)}(\mathscr{N})$ complementary to the radical, then $Y \oplus \mathscr{Z}(\mathscr{N})=\mathscr{H}$ will be an ideal in $\mathscr{N}$, and we can write $\mathscr{N}=$ $C(\mathscr{H})+\mathscr{H}$, with $C(\mathscr{H})$ the centralizer of $\mathscr{H}$ in $\mathscr{N}$. Further

$$
C(\mathscr{H}) \cap \mathscr{H}=\mathscr{Z}(\mathscr{N})=\mathscr{Z}(\mathscr{H})=\mathscr{Z}(C(\mathscr{H})),
$$

so $\mathscr{N}$ is the "central product" of $\mathscr{H}$ and $C(\mathscr{H})$. Furthermore $\mathscr{Z}^{(2)}(C(\mathscr{H}))=\mathscr{Z}\left(\mathscr{Z}^{(2)}(\mathscr{N})\right)$ will be abelian. We will call a lie algebra with abelian second center $H$-reduced, since it is then not possible to factor off an Heisenberg group as above. Thus any $\mathscr{N}$ with one-dimensional center can be factored into the central product of a Heisenberg Lie algebra and an $H$-reduced algebra.

I claim that such a decomposition is unique up to isomorphism, in the sense that if have two central product decompositions of $\mathscr{N}$ into Heisenberg and $H$-reduced pieces, there is an automorphism of $\mathscr{N}$ taking one decomposition to the other. Indeed, two such decompositions result from choosing two different complements $Y$ and $Y^{\prime}$ to $\mathscr{Z}\left(\mathscr{Z}^{(2)}(\mathscr{N})\right.$. Let the resulting decompositions of of $\mathscr{N}$ be written in the form

$$
\begin{aligned}
\mathscr{N} & =C\left(\mathscr{F}^{(2)}(\mathscr{N})\right) \oplus Y \oplus X \\
& =C\left(\mathscr{F}^{(2)}(\mathscr{N})\right) \oplus Y^{\prime} \oplus X^{\prime}
\end{aligned}
$$

Here $X$ commutes with $Y$ and $X^{\prime}$ commutes $Y^{\prime}$. We may represent an element of $Y^{\prime}$ in the form 


$$
y^{\prime}=y+A(y)
$$

where $A \in \operatorname{Hom}\left(Y, \mathscr{z}\left(\mathscr{Z}^{(2)}(\mathscr{N})\right)\right)$

Similarly, we may represent an element of $X^{\prime}$ in the

$$
x^{\prime}=x+B(x)+C(x)
$$

where $B \in \operatorname{Hom}(X, Y)$ and $C \in \operatorname{Hom}\left(X, C\left(\mathscr{Z}^{(2)}(\mathscr{N})\right)\right.$. We will show that, given $A$ we can find $B$ and $C$ such that the direct sum of $y \rightarrow y^{\prime}, x \rightarrow x^{\prime}$ and the identity on $C\left(\mathscr{Z}^{(2)}(\mathscr{N})\right)$ will be an automorphism of $\mathscr{N}$. We will actually choose $\left.C \in \operatorname{Hom}\left(X, \mathscr{Z}\left(\mathscr{Z}^{(2)} \mathscr{N}\right)\right)\right)$.

The condition that $X^{\prime}$ and $Y^{\prime}$ commute is

$$
\begin{aligned}
0=\left[x^{\prime}, y^{\prime}\right] & =[x+B(x)+C(x), y+A(y)] \\
& =[x, A(y)]+[B(x), y]
\end{aligned}
$$

The bracket $[x, A(y)]$ is, as a function of $y$, an element of $\operatorname{Hom}(Y$, $\mathscr{Z}(\mathscr{N}))$. Since bracket makes $Y$ isomorphic to $\operatorname{Hom}(Y, \mathscr{K}(\mathscr{N}))$, there is a unique choice of $B(x)$ so that we do have $[x, y]=0$. It remains then to choose $C$ so that the indicated map will be an automorphism of $\mathscr{N}$. Since $C$ is to take values in $\mathscr{Z}\left(\mathscr{Z}^{(2)}(\mathscr{N})\right)$, the only brackets to worry about are those of $X^{\prime}$ with itself. We compute

$$
\begin{aligned}
{\left[x_{1}^{\prime}, x_{2}^{\prime}\right]=} & {\left[x_{1}+B\left(x_{1}\right)+C\left(x_{1}\right), x_{2}+B\left(x_{2}\right)+C\left(x_{2}\right)\right] } \\
= & {\left[x_{1}, x_{2}\right]+\left[B\left(x_{1}\right), B\left(x_{2}\right)\right] } \\
& +\left[x_{1}, C\left(x_{2}\right)\right]-\left[x_{2}, C\left(x_{1}\right)\right]
\end{aligned}
$$

The bracket $\left[B\left(x_{1}\right), B\left(x_{2}\right)\right]$ is an alternating $\mathscr{F}(\mathscr{N})$-valued bilinear form on $X$. Since, by construction of $X$ we have

$$
\mathscr{Z}\left(\mathscr{Z}^{(2)}(\mathscr{N})\right) / \mathscr{Z}(\mathscr{N}) \simeq \operatorname{Hom}(X, \mathscr{Z}(\mathscr{N}))
$$

we see that the bracket $\left.] x_{1}, C\left(x_{2}\right)\right]$ is an arbitrary $\mathscr{Z}(\mathscr{N})$-valued bilinear form on $X$, and $\left[x_{1}, C\left(x_{2}\right)\right]-\left[x_{2}, C\left(x_{1}\right)\right]$ is its antisymmetrization. Thus $C$ may be chosen so that $\left[x_{1}^{\prime}, x_{2}^{\prime}\right]=\left[x_{1}, x_{2}\right]$, and our claim concerning uniqueness of the decomposition

$$
\mathscr{N}=\mathscr{H}+C(\mathscr{H})
$$

into Heisenberg and $H$-reduced factors is proven.

Now return to our split HAT Lie algebra $\mathscr{N}=S_{0} \oplus S^{*}$. We have computed above that $\mathscr{Z}^{(2)}(\mathscr{N})$ is the sum $\eta\left(S_{0}\right) \oplus\left(S_{0}^{2}\right)^{\perp}$. On the other hand, the centralizer of $\eta\left(S_{0}\right)$ in $S^{*}$ is $\eta\left(S_{0}\right)^{\perp}$. Thus $\mathscr{z}^{(2)}(\mathscr{N})$ is nonabelian if and only if $\left(S_{0}^{2}\right)^{\perp} \nsubseteq \eta\left(S_{0}\right)^{\lrcorner}$, that is, if and only if $\eta\left(S_{0}\right) \nsubseteq S_{0}^{2}$. On the other hand, write $S_{0}=X+\widetilde{S}$, where $X$ is a complement to $S_{0}^{2} \cap \eta\left(S_{0}\right)$ in $\eta\left(S_{0}\right)$, and $S_{0}^{2}=\widetilde{S}_{0}^{2} \supseteqq \eta\left(\widetilde{S}_{0}\right)$. Then we can write 


$$
\mathscr{N}=\left(\widetilde{S}_{0} \oplus \widetilde{S}^{*}\right) \oplus\left(X \oplus X^{*}\right)
$$

and $X \oplus X^{*} \oplus \mathscr{Z}(\mathscr{N})$ is Heisenberg, while $\tilde{\mathscr{N}}=\widetilde{S}_{0} \oplus \widetilde{S}^{*}$ is $H$-reduced by the above remarks and the construction of $S_{0}$. By the essential uniqueness of such a decomposition, part (a) of the proposition is reduced to part (b). But by our analysis above, when $\eta\left(S_{0}\right) \subseteq S_{0}^{2}$, we have

$$
\eta\left(S_{0}\right) \simeq\left(\mathscr{\varkappa}^{(2)}(\mathscr{N})+\mathscr{N}^{(2)}\right) / \mathscr{N}^{(2)},
$$

so that $\eta\left(S_{0}\right)$ is determined modulo $\mathscr{N}^{(2)}$ by $\mathscr{N}$. Therefore if $\mathscr{N} \simeq$ $S_{0}^{\prime} \oplus S^{\prime}$ is another presentation of $\mathscr{N}$ as HAT Lie algebra, then $\eta\left(S_{0}\right)=\eta\left(S_{0}^{\prime}\right)$ modulo $\mathscr{N}^{(2)}=\eta\left(S_{0}\right)^{\perp}=\eta\left(S_{0}^{\prime}\right)^{\perp}$. Hence $\eta\left(S_{0}\right)+S^{\prime *}=$ $C\left(\mathscr{N}^{(2)}\right)$, and therefore $\mathscr{N} \simeq S_{0} \oplus S^{*}$, as asserted, and so $S_{0}$ and $S_{0}^{\prime}$ induce the same algebra of endomorphisms on $S^{\prime *}$. This completes the proposition.

Remarks. (a) I am grateful to Richard Penny for pointing out an error in the original formulation and proof of Proposition 2.9.

(b) Bracket in the split HAT Lie algebra $\mathscr{N}=S_{0} \oplus S^{*}$ induces a symplectic $\mathscr{Z}(\mathscr{N})$-valued from on $C\left(\mathscr{N}^{(2)}\right) / \mathscr{N}^{(2)}$, and we may choose as complement to $\eta\left(S_{0}\right)$ the inverse image in $C\left(\mathscr{N}^{(2)}\right)$ of any isotropic complement to $\eta\left(S_{0}\right)$ in $C\left(\mathscr{N}^{(2)}\right) / \mathscr{N}^{(2)}$. It is clear that the automorphism subgroup of $\mathscr{N}$ leaving $S_{0}$ pointwise fixed acts transitively on the set of such complements.

(c) It should be pointed out that a split HAT group can also be represented as a nonsplit HAT group for a different algebra. Thus if $S_{0}$ is generated by $x$ with $x^{3}=0$, we can construct the 5dimensional split HAT group $\mathscr{N}=S_{0} \oplus S^{*}$. This will have basis $\left\{x, x^{2}, f_{1}, f_{2}, f_{3}\right\}$, where $f_{1}, f_{2}, f_{3}$ is the basis of $S^{*}$ dual to $\left\{1, x, x^{2}\right\}$. Thus $f_{1}$ spans the center of $\mathscr{N}$, and

$$
\left[x, f_{2}\right]=\left[x^{2}, f_{3}\right]=f_{1} \text {, and }\left[x^{2}, f_{3}\right]=f_{1},
$$

other brackets being zero. Thus $\left\{f_{1}, f_{2}, x^{2}\right\}$ span an abelian ideal $\mathscr{M}$, and $\mathscr{N} / \mathscr{C}$ is abelian, being spanned by $\left\{x, f_{3}\right\}$. Further, the action of $\mathscr{N} / \mathscr{M}$ on $\mathscr{C}$ is by the algebra generated by $x$ and $y$, with $x^{2}=x y=y^{2}=0$, so $\mathscr{N}$ is an nonsplit HAT group for this algebra. Of course, the extent to which this phenomenon can occur is limited by Proposition $2.6(\mathrm{~b})$.

3. Smooth harmonic analysis. To relate harmonic analysis on nilpotent groups to classical problems, it seems necessary to go beyond $L^{2}$ analysis and consider questions of smoothness. The argument of this section is that the smooth representation theory of nilpotent groups is nice and deserving of some development. We 
divide our attention between writing down basic definitions, proving: certain facts, and formulating some problems.

Let $V$ be a real vector space. We recall the definition of the Schwartz space $\mathscr{S}(V)$. Let $\mathscr{P} \mathscr{D}(V)$ be the polynomial coefficient differential operators on $V$. Contained in $\mathscr{P} \mathscr{D}(V)$ are $\mathscr{P}(V)$, the polynomials on $V$, and $\mathscr{D}(V)$, the constant coefficient differential operators, and $\mathscr{P} \mathscr{D}$, as a linear space, is the tensor product of $\mathscr{P}$ and $\mathscr{D}$. The definition of $\mathscr{S}$ is

$$
\mathscr{S}(V)=\left\{f \in C^{\infty}(V): \sup \left\{T f(x): x \in \boldsymbol{R}^{n}\right\}<\infty \text {, all } T \in \mathscr{P} \mathscr{D}(V)\right\} .
$$

Thus $\mathscr{S}$ is defined by means of $\mathscr{P} \mathscr{D}$.

By a polynomial diffeomorphism of $V$ we mean a biregular map, in the sense of algebraic geometry, from $V$ to $V$. Concretely, $\phi: V \rightarrow V$ is a polynomial diffeomorphism if $\phi$ and $\phi^{-1}$ are given in any linear coordinate system by polynomials. Another alternative is to regard $\phi$ as an automorphism of $\mathscr{P}$.

Let $\phi$ be a polynomial diffeomorphism on $\boldsymbol{R}^{n}$, and let $p \in \mathscr{P}\left(\boldsymbol{R}^{n}\right)$. Define $\phi^{*}(p)=\operatorname{Ad} \phi(p)=p \circ \phi$. Similarly, if $\nu=\sum_{i=1}^{n} p_{i}\left(\partial / \partial x_{i}\right)$ is a vector field, define $\operatorname{Ad} \phi(\nu)$ by $\operatorname{Ad} \phi(\nu)(f)=\left(\nu\left(f \circ \phi^{-1}\right)\right) \circ \phi$. We compute

$$
\operatorname{Ad} \phi(\nu)=\sum_{i, j}\left(p_{i} \circ \phi\right) a_{i j} \frac{\partial}{\partial x_{j}}
$$

where $\left\{a_{i j}\right\}$ is the matrix inverse to the Jacobian $\left\{\partial \phi / \partial x_{i}\right\}$ of $\phi$. We recall the relation

$$
\sum_{i=1}^{n}\left(\frac{\partial \phi_{j}^{-1}}{\partial x_{i}} \circ \phi\right) \frac{\partial \phi_{i}}{\partial x_{k}}=\delta_{j k} .
$$

It follows that $\operatorname{det}\left\{\partial \phi_{j} / \partial x_{i}\right\}$ is both a polynomial and the inverse of a polynomial, hence constant. Therefore the $a_{i j}$ are polynomial functions also. Thus Ad $\phi$ defines an automorphism of $\mathscr{P}$ and of the polynomial coefficient vector fields. Since $\mathscr{P} \mathscr{D}$ is generated by these spaces, we see Ad $\phi$ (extended in the obvious way) defines an automorphism of $\mathscr{P} \mathscr{D}$. Consequently, $\phi^{*}: f \rightarrow f \circ \phi$ defines an automorphism of $\mathscr{S}(V)$ (as a topological vector space). In other words, $\mathscr{S}(V)$ is a space of functions attached invariantly not to the rigid linear structure of $V$ but to the structure of $V$ as affine algebraic variety. (In fact, it is attached to an even looser structure, preserved by what one might call the "diffeomorphisms of polynomial growth," but the affine structure is flexible enough for our present purposes.)

Let $N$ be a (connected, simply connected) nilpotent Lie group. The exponential map exp: $\mathfrak{R} \rightarrow N$ is a diffeomorphism with log: as inverse. We define $\mathscr{S}(N)$ to be the pullback to $N$ by log of $\mathscr{S}(\mathfrak{R})$. 
In the coordinates on $N$ induced by exp, left and right translation are both polynomial diffeomorphisms, so $N$ acts continuously to the right and to the left on $\mathscr{S}(N)$. Also it is not difficult to check that $\mathscr{S}(N)$ is closed under convolution, and that it becomes a topological algebra with convolution as multiplication.

Let $M \cong N$ be a connected subgroup. Choose elements $\left\{x_{i}\right\}_{i=1}^{l} \subseteq \mathfrak{R}$ such that if $\mathfrak{M}_{j}$ is the span of $\mathfrak{M}$ and the $x_{i}$ for $i \leqq j$, then $\mathfrak{M}_{j}$ is a Lie subalgebra of $\mathfrak{N}$ and an ideal in $\mathfrak{M}_{j+1}$, and such that $\mathfrak{M}_{i}=\mathfrak{N}$. Then define

$$
X=\left\{\exp t_{l} x_{l} \cdots \exp t_{1} x_{1}: t_{i} \in \boldsymbol{R}\right\} .
$$

It is well-known that $N=X \cdot M$, and group multiplication in $N$ takes $X \times M$ diffeomorphically to $N$. Thus the natural projection $N \rightarrow$ $N / M$ takes $X$ diffeomorphically to $N / M$. We call such an $X$ a standard smooth cross-section to $M$ in $N$. We parametrize $X$, and hence $N / M$ by means of the coordinates $t_{i}$ in its definition. We define $\mathscr{S}(N / M)$ to be the corresponding Schwartz space. It is wellknown that a choice of a sequence other than $x_{i}$ leads to a reparametrization of $N / M$ differing from the first by a polynomial diffeomorphism of $\boldsymbol{R}^{l}$. Thus $\mathscr{S}(N / M)$ is well-defined. We also remark that if $\mathfrak{M}$ is an ideal in $\mathfrak{R}$, and $U \subseteq \mathfrak{N}$ is a linear complement to $\mathfrak{M}$, then $\exp U$ also parametrizes $N / M$ and using linear coordinates on $U$ leads to the same Schwartz space on $N / M$. In particular, our definition of $\mathscr{S}(N / M)$ in the case $M=\{1\}$ is consistent with our previous definition of $\mathscr{S}(N)$.

Slightly more generally, take a unitary character $\psi$ of $M$. This will define a line bundle over $N / M$. The sections of this line bundle can be thought of as functions on $N$ such that $f(n m)=\psi(m)^{-1} f(n)$ for $n \in N$ and $m \in M$. We define $\mathscr{S}(N / M, \psi)$ to be those functions $f$ which transform as above and whose restriction to $X$ belongs to $\mathscr{S}(X)$. Here a reparametrization leads to a polynomial diffeomorphism composed with multiplication by a function of the form $e^{i p(x)}$ where $p$ is a polynomial. The following easily verified lemma shows that in this case too the definition of $\mathscr{S}(N / M, \psi)$ is unambiguous.

Lemma 3.1. Let $V$ be a real vector space, let $\phi$ be a function on $V$ all of whose derivatives have polynomial growth. Then $f \rightarrow \phi f$, the operation of multiplication by $\phi$, defines a continuous endomorphism of $\mathscr{S}(V)$.

We may define $L^{2}(N / M, \psi)$ similarly to $\mathscr{S}(N / M, \psi)$. It is the space of functions $f$ on $N$ such that $f(n m)=\psi(m) f(a)$ as before, and such that $f_{\mid X} \in L^{2}(X)$. (As always, one identifies functions which are equal almost everywhere on $X$.) Evidently, $\mathscr{S}(N / M, \psi)$ is con- 
tained in $L^{2}(N / M, \psi)$ as a dense subspace. Moreover, $L^{2}(N / M, \psi)$ is the space of the unitary induced representation $\operatorname{ind}_{M}^{N} \psi$, and $\mathscr{S}(N / M, \psi)$ is an invariant subspace. The unitary decomposition of $L^{2}(N / M, \psi)$ was more or less described by Kirillov. I would like to draw attention to the following.

Problem. Find a "smooth decomposition" for the "smoothly induced" representation of $N$ on $\mathscr{S}(N / M, \psi)$.

The extreme cases of this problem are of course the case $M=1$, the regular representations on the one hand, and on the other, the case when $\operatorname{ind}_{M}^{N} \psi$ is irreducible. We will treat the irreducible case more or less completely. We will also give some attention to the regular representation, but our discussion will be far from complete. A full treatment would involve putting a smooth structure on the unitary dual $\hat{N}$ of $N$, and on the "operator algebra bundle" over it, and then proving a version of the "Paley Wiener theorem" describing the sections of this bundle defined by $\mathscr{S}(N)$.

One fact which is basic for all $\operatorname{ind}_{M}^{N} \psi$ is this one:

Proposition 3.2. Consider the map $P_{M, \psi}=P$ defined on $\mathscr{S}(N)$ by $\operatorname{Pf}(n)=\int_{M} \psi(m) f(n m) d m$. Then $P$ defines a continuous open surjection from $\mathscr{S}(N)$ to $\mathscr{S}(N / M, \psi)$.

Proof. This is well-known and easy when $N$ is abelian. By proper choice of coordinates, we can make this projection look like the abelian case. Indeed, let $X$ be a standard cross-section to $M$ in $N$. The map $\mu: X \times M \rightarrow N$ defined by multiplication in $N$ defines an isomorphism $\mu^{*}: \mathscr{S}(N) \cong \mathscr{S}(X \times M)$. On $\mathscr{S}(X \times M)$ we may define $\widetilde{P}$ by $\widetilde{P} f(x, m)=\int_{M} f\left(x, m m^{\prime}\right) \psi\left(m^{\prime}\right) d m^{\prime}$. If we use canonical coordinates in $M$, then $\widetilde{P}$ has precisely the form of the abelian projection. Since $\mu^{*} \circ P=\widetilde{P} \circ \mu^{*}$, the proposition follows.

Now focus on irreducible representations. One of Kirillov's original results [8] says that if coordinates from some standard cross-section to $M$ are used to identify $\mathscr{S}(N / M, \psi)$ to $\mathscr{S}\left(R^{n}\right)$, then the action of $\mathscr{U}(\mathfrak{N})$, the universal enveloping algebra of $\mathfrak{R}$ on $\mathscr{S}(N / M, \psi)$ transfers to the action of all of $\mathscr{P} \mathscr{D}\left(\boldsymbol{R}^{n}\right)$ on $\mathscr{S}\left(\boldsymbol{R}^{n}\right)$. This fact has been siezed on by the universal enveloping algebraists, but its analytic consequences have not been emphasized. Let us state the most obvious one.

Proposition 3.3 (Kirillov). If an irreducible unitary representation $\rho$ of $N$ is realized as ind $\operatorname{ind}_{m}^{N} \psi$, then $C^{\infty}(\rho)$, the space of smooth vectors for $\rho$, is identified to $\mathscr{S}(N / M, \psi)$. 
Consider the operators on $L^{2}(N / M, \psi)$. These may be realized as kernel operators, in general with distributional kernels, belonging to $\mathscr{S}^{*}\left((N \times N) /(M \times M), \psi \times \psi^{*}\right)$. If the operator $T$ has a kernel which is a continuous function $K(x, y)$ with $x, y \in N$ and $K\left(x m, y m^{\prime}\right)=$ $\psi\left(m^{-1} m^{\prime}\right) K(x, y)$ for $m, m^{\prime} \in M$, then for $\phi \in \mathscr{S}(N / M, \psi)$, we have

$$
T(\phi)(x)=\int_{N / M} K(x, y) \phi(y) d y .
$$

Note that for a fixed $x$, the function $K(x, y) \phi(y)$ is right invariant by $M$, so the integral is well-defined. Note also that right and left multiplication of $T$ by $\rho(N)$ corresponds to translation in the first and second variables respectively. That is, the kernel of $\rho\left(n_{1}\right) T \rho\left(n_{2}\right)$ is $K\left(n_{1}^{-1} x, n_{2} y\right)$. Thus in any standard coordinate system on $N \times$ $N / M \times M$, the left and right actions of $\mathscr{C}(\mathfrak{R})$ together generate all polynomial coefficient differential operators, by Kirillov's result.

We call an operator on $L^{2}(N / M, \psi)$ smooth if it remains bounded when preceded and followed by arbitrary elements of $\rho(\mathscr{C}(\Re))$. We denote the smooth operators by $C^{\infty}(\mathscr{L}(\rho))$. By our remark above on the action of $\mathscr{U}(\mathfrak{N})$ on kernels, we see that $C^{\infty}(\mathscr{L}(\rho))$ is precisely those operators whose kernels belong to $\mathscr{S}\left((N \times N) /(M \times M), \psi \times \psi^{*}\right)$. It is clear that if $f \in \mathscr{S}(N)$, then $\rho(f) \in C^{\infty}(\mathscr{L}(\rho))$.

THEOREM 3.4. The map $\rho: \mathscr{S}(N) \rightarrow C^{\infty}(\mathscr{L}(\rho))$ is surjective.

REMARK. Since this result is the most delicate of the paper, we write down most of the proof, although it is of a standard type and rather clumsy. We proceed quite constructively. Presumably general methods can be developed to prove results of this nature. Also the Paley-Wiener theorem suggested above for the regular representation would imply this theorem very quickly. Reciprocally, an argument like this one might imply a weak version of PaleyWiener, covering representations "in general position."

Proof. We will actually prove a somewhat more precise result, which we now formulate. By a basis of $\mathscr{S}(N)$, or other similar space, we mean a linearly independent sequence $\left\{e_{i}\right\}_{i=1}^{\infty} \subseteq \mathscr{S}(N)$ such that:

$$
\mathscr{S}(N)=\left\{\sum_{i=1}^{\infty} a_{i} e_{i}: \sum_{i=1}^{\infty}\left|a_{i}\right| i^{n}<\infty \text { all } n\right\} .
$$

and the obvious seminorms define the topology on $\mathscr{S}(N)$. It is known that bases exist. For $\mathscr{S}(\boldsymbol{R})$, for example, the Hermite functions give a nice example. Let us note also that if $\left\{\boldsymbol{e}_{i}\right\}_{i=1}^{\infty}$ is a basis for $\mathscr{S}(N)$ and $\left\{f_{i}\right\}_{i=1}^{\infty}$ is a basis for $\mathscr{S}(M)$, then $\left\{e_{i} \otimes f_{j}\right\}_{i, j=1}^{\infty}$ 
may be renumbered to form a basis for $\mathscr{S}(N \times M) \cong \mathscr{S}(N) \otimes \mathscr{S}(M)$. For example, put $e_{i} \otimes f_{j}=g_{k}$ where $k=(1 / 2)(i+j-1)(i+j-2)+i$, then $\left\{g_{k}\right\}_{k=1}^{\infty}$ is a basis for $\mathscr{S}(N \times M)$.

We will show

THEOREM 3.4 a. The map $\rho: \mathscr{S}(N) \rightarrow C^{\infty}(\mathscr{L}(\rho))$ is onto. Moreover, $\operatorname{ker} \rho$ is complemented in $\mathscr{S}(N)$. Specifically, there is a sequence $\left\{f_{i}\right\}_{i=1}^{\infty} \subseteq \mathscr{S}(N)$ such that $\left\|f_{i}\right\|$ grows at most polynomially in $i$ for any continuous seminorm on $\mathscr{S}(N)$, and $\left\{\rho\left(f_{i}\right)\right\}_{i=1}^{\infty}$ is a basis for $C^{\infty}(\mathscr{L}(\rho))$.

Our argument proceeds by induction as usual. We may as well assume that $\rho$ is locally faithful. Then $\operatorname{dim} \not{Z}(N)=1$ and $\rho$ is nontrivial on $\mathscr{z}(N)$. Let $z_{a}$, with $a \in \boldsymbol{R}$, denote the elements of $\not{z}(N)$, and normalize this parametrization so that $\rho\left(z_{a}\right)=e^{2 \pi i a}$. Choose a one-parameter subgroup $X$ of $\mathscr{F}^{2}(N)$, the second center of $N$. Elements of $X$ will be $x_{b}, b \in \boldsymbol{R}$. Let $N_{1}$ be the centralizer of $X$ in $N$. Then $\operatorname{dim} N_{1}=\operatorname{dim} N-1$ and $N_{1}$ is normal in $N$. Choose a oneparameter group $Y$ complementary to $N_{1}$ in $N$. Let $Y$ be parametrized by $y_{c}, c \in \boldsymbol{R}$. We suppose the parametrizations are normalized so that $\operatorname{Ad} y_{c}\left(x_{b}\right)=y_{c} x_{b} y_{c}^{-1}=x_{b} z_{b c}$. Together the groups $X, Y$, and $\mathscr{Z}(N)$ form a 3-dimensional Heisenberg group $H$ such that $H \cap N_{1} \subseteq$ $\mathscr{Z}\left(N_{1}\right)$ and $H \cdot N_{1}=N$.

We may realize $\rho$ as $\operatorname{ind}_{M}^{N} \psi$, where $M \subseteq N_{1}$. Necessarily, $H \cap$ $N_{1} \subseteq M$. Of course, $\psi\left(z_{a}\right)=e^{2 \pi i z}$, and we may assume that $\psi\left(x_{b}\right)=1$ for all $b$. If we introduce appropriate standard coordinates on $N / M$, then we can consider $\rho$ as realized on functions on $\boldsymbol{R} \times \boldsymbol{R}^{k}$, such that the following formulas hold. Here $t \in \boldsymbol{R}$, and $v \in \boldsymbol{R}^{k}$.

$$
\begin{aligned}
& \rho\left(y_{c}\right) f(t, v)=f(t-c, v) \\
& \rho\left(x_{b}\right) f(t, v)=e^{2 \pi i t b} f(t, v) \\
& \rho(n) f(t, v)=\sigma_{0}\left(\operatorname{Ad} y_{t}^{-1}(n)\right) f(t, v),
\end{aligned}
$$

for $n \in N_{1}$, where $\sigma_{0}$ is the representation $\operatorname{ind}_{M}^{N} \psi$, and $\sigma_{0}(n)$ acts only on the $\boldsymbol{R}^{k}$-variables.

Let $\mathfrak{U} \subseteq \mathfrak{N}_{1}$ be a complement to $\mathfrak{S} \cap \mathfrak{R}_{1}$, and let $U=\exp \mathfrak{U}$. Then $U$ is a cross-section to $H \cap N_{1}$ in $N_{1}$. Choose a Lebesgue measure on $U$ so that $d u d b d a$ is Haar measure on $N_{1}$. For $f \in \mathscr{S}\left(N_{1}\right)$, define $p(f) \in \mathscr{S}(U)$ by

$$
p(f)(u)=\int f\left(u x_{b} z_{a}\right) e^{2 \pi i a} d a d q .
$$

Proposition 3.2 says $p$ is a continuous projection of $\mathscr{S}\left(N_{1}\right)$ onto $\mathscr{S}(U)$. Moreover $\sigma_{0}(f)=\sigma_{0}(p(f) d u)$, where the right hand side means the natural extension of $\sigma_{0}$ to measures. 
Write $\operatorname{Ad}^{*} y_{c}\left(\sigma_{0}\right)=\sigma_{c}$, so that $\sigma_{c}\left(\operatorname{Ad}^{*} y_{c}(f)\right)=\sigma_{0}(f)$. If we define $p_{c}(f)(u)=\int f\left(u x_{b} z_{a}\right) e^{2 \pi i(a-b c)} d a d b$, then $\sigma_{c}(f)=\sigma_{c}\left(p_{c}(f) d u\right)$. Let us compute $p_{c}\left(\operatorname{Ad}^{*} y_{c}(f)\right)$. We may write $\operatorname{Ad} y_{c}\left(u x_{b} z_{a}\right)=A_{c}(u) \lambda_{c}(u) x_{b} z_{a+b c}$. In this formula $A_{c}$ is the map of $U$ into itself induced by identifying $U \cong N_{1} /\left(H \cap N_{1}\right)$ and taking the quotient of Ad $y_{c}$. The term $\lambda_{c}(u)$ is defined by $\lambda_{c}(u)=\operatorname{Ad} y_{c}(u) A_{c}(u)^{-1}$. The map log $\circ \lambda_{c} \circ$ exp: $\mathfrak{u} \rightarrow$ $\mathfrak{S} \cap \mathfrak{N}_{1}$ is linear. We compute

$$
\begin{aligned}
p_{c}\left(\operatorname{Ad}^{*} y_{c}(f)\right) & =\int f\left(\operatorname{Ad} y_{c}^{-1}\left(u x_{b} z_{a}\right)\right) e^{2 \pi i(a-b c)} d a d b \\
& \left.=\int f\left(A_{c}^{-1}(u) \lambda_{-c}(u) x_{b} z_{a-b c}\right) e^{2 \pi i(a-b c}\right) d a d b \\
& =\int f\left(A_{c}^{-1}(u) \lambda_{-c}(u) x_{b} z_{a}\right) e^{2 \pi i a} d a d b \\
& =\int \psi^{-1}\left(\lambda_{-c}(u)\right) f\left(A_{c}^{-1}(u) x_{b} z_{a}\right) e^{2 \pi i a} d a d b \\
& =\psi^{-1}\left(\lambda_{-c}(u)\right) p_{0}(f)\left(A_{c}^{-1}(u)\right) \\
& =\phi_{c}(u) A_{c}^{*} p_{0}(f)(u),
\end{aligned}
$$

where we have set $\phi_{c}(u)=\psi^{-1}\left(\lambda_{-c}(u)\right)$. Combining this with previous formulas, we have

$$
\sigma_{c}\left(\phi_{c} A_{c}^{*}\left(p_{0}(f)\right)\right)=\sigma_{c}(f) .
$$

In the coordinates on $U$ derived from linear coordinates on $\mathfrak{U}$ via exp, the transformation $A_{c}$ is linear and depends polynomially on $c$. Similarly, $\phi_{c}$ is a unitary character depending polynomially on $c$. Therefore we see we may find a cofinal family of seminorms \|\| on $\mathscr{S}(U)$ such that $\left\|\phi_{c} A_{c}^{*}(g)\right\| \leqq q(c)\|g\|$ for $g \in \mathscr{S}(U)$ and some polynomial $q$ in $y$. Of course, $q$ depends on \|\| .

Choose any $w \in \mathscr{S}(\boldsymbol{R})$ with $\int_{\boldsymbol{R}} w d t=1$. For $g \in \mathscr{S}(U)$ and $e \in$ $\mathscr{S}(\boldsymbol{R})$, put

$$
F(g, e)\left(u x_{b} z_{a}\right)=\left(\int_{R} \phi_{c}(u) A_{c}^{*}(g)(u) e(c) e^{2 \pi i c b} d c\right) e^{-2 \pi i a} w(a) .
$$

A straightforward application of the Fourier inversion formula for $\boldsymbol{R}$ yields the result

$$
p_{c}(F(g, e))=e(c) \phi_{c} A_{c}^{*}(g) .
$$

Hence

$$
\sigma_{c}(F(g, e))=e(c) \sigma_{0}(g)
$$

Therefore, finally

$$
\rho(F(g, e))(f(t, v))=e(t) \sigma_{0}(g)(f(t, v)) .
$$


Moreover, our estimates on $\phi_{c} A_{c}^{*}(g)$ show $F(g, e)$ depends continuously on $g$ and $c$.

By induction, we may assume there is a sequence $\left\{f_{i}\right\}_{i=1}^{\infty} \subseteq \mathscr{S}\left(N_{1}\right)$ such that $\left\|f_{i}\right\|$ has polynomial growth in $i$ for any seminorm and $\left\{\sigma_{0}\left(f_{i}\right)\right\}_{i=1}^{\infty}$ is a basis for $C^{\infty}\left(\mathscr{L}\left(\sigma_{0}\right)\right)$. In our coordinates then, the $\sigma_{0}\left(f_{i}\right)$ are a basis for the operators on $\mathscr{S}\left(\boldsymbol{R}^{k}\right)$ given by Schwartz kernels. These may also be regarded as the operators on $\mathscr{S}\left(\boldsymbol{R} \times \boldsymbol{R}^{k}\right)$ which are independent of the $\boldsymbol{R}^{k}$-variable. We may also regard $\mathscr{S}(\boldsymbol{R})$ as operating on $\mathscr{S}\left(\boldsymbol{R} \times \boldsymbol{R}^{k}\right)$, by multiplication operators depending only on the $\boldsymbol{R}$-variable. Let $\left\{e_{i}\right\}_{i=1}^{\infty}$ be a basis for $\mathscr{S}(\boldsymbol{R})$. As we saw earlier, we may renumber the set $e_{i} \otimes \sigma_{0}\left(f_{j}\right)$ so that it forms a basis for all smooth kernel operators which leave each subspace $t \times \boldsymbol{R}^{k}$-invariant, that is, all smooth kernel operators commuting with $\rho\left(x_{b}\right)$ for all $b$. We see, however, from our formulas above that

$$
e_{i} \otimes \sigma_{0}\left(f_{j}\right)=\rho\left(F\left(p_{0}\left(f_{j}\right), e_{i}\right)\right)
$$

and it is clear from the estimates above that $\left\|F\left(p_{0}\left(f_{j}\right), e_{i}\right)\right\|$ grows at most polynomially in $i$ and $j$ for any continuous seminorm \|\| on $\mathscr{S}\left(N_{1}\right)$.

Thus we have shown $\rho$ maps $\mathscr{S}\left(N_{1}\right)$ onto the smooth kernel operators commuting with $\rho\left(x_{b}\right)$. It remains only to throw in the translations along the $X$-spectrum (i.e., the $R$-variable). This is done in straightforward analogy with the above, using the oneparameter group $Y$. The details are left to the reader.

COROLlaRY 3.4.1. $\rho(\mathscr{S}(N))$ acts algebraically irreducibly on $C^{\infty}(\rho)$ for $\rho \in \hat{N}$.

COROLLARY 3.4.2. Let $\rho \in \hat{N}$ and let $\sigma$ be any unitary representation of $N$. Let $\mathscr{H}_{\rho}$ and $\mathscr{H}_{\sigma}$ be the spaces of these representations. Let $T: C^{\infty}\left(\mathscr{H}_{\rho}\right) \rightarrow \mathscr{H}_{\sigma}$ be any intertwining map for the action of $\mathscr{S}(N)$. Then $T$ is isometric up to multiples.

Proof. Let $v_{1}$ and $v_{2}$ be two orthogonal smooth vectors in $\mathscr{H}_{\rho}$. Let $P$ be an orthogonal projection onto the line through $v_{1}$, so $P v_{1}=v_{1}$ and $P v_{2}=0$. Then $P \in C^{\infty}(\mathscr{L}(\rho))$, so $P=\rho(f)$ for some $f \in \mathscr{S}(N)$. Then also $P=\rho\left(f * f^{*}\right)$, where $f^{*}(n)=\bar{f}\left(n^{-1}\right)$, the ${ }^{-}$indicating complex conjugation, and $f * f^{*}$ means the convolution of $f$ and $f^{*}$. Put $f * f^{*}=g$. Since $T$ intertwines $C^{\infty}\left(\mathscr{H}_{\rho}\right)$ and $\mathscr{H}_{o}$, we have $T v_{1}=T\left(\rho(g) v_{1}\right)=\sigma(g)\left(T v_{1}\right)$, while $\sigma(g)\left(T v_{2}\right)=0$. Therefore $T v_{1}$ and $T v_{2}$ are in distinct eigenspaces of the positive operator $\sigma(g)$ and so are orthogonal. Thus $T$ preserves orthogonality, so it must be a multiple of an isometry. 
REMARK. Poulsen [13] has proved a similar result for general Lie groups. A result of this type greatly shortens arguments such as given in [7] and elsewhere.

Corollary 3.4.3. If $\rho \in \hat{N}$ and $\rho \cong \operatorname{ind}_{k}^{N} \psi$, then there is in $C^{\infty}(\rho)^{*}$ precisely one eigenvector with eigencharacter $\psi$ for $M$.

We now pass to the consideration of some aspects of the regular representation, mainly its central decomposition. Our proofs in this discussion will be very sketchy. As noted at the beginning of this section, $\mathscr{S}(N)$ is an algebra under convolution. However, one may convolve a Schwartz function $f$ with a distribution $D \in \mathscr{S}(N)^{*}$. The result will be a smooth function which may not, however, belong to $\mathscr{S}(N)$ because it may not die fast at $\infty$. In explicit terms, one has the formulas

$$
\begin{aligned}
& (D * f)(n)=D\left(L_{n} \breve{f}\right) \quad \text { and } \\
& (f * D)(n)=D\left(R_{n^{-1}} \check{f}\right)
\end{aligned}
$$

where $\breve{f}(n)=f\left(n^{-1}\right)$, and $L_{n} f\left(n^{\prime}\right)=f\left(n^{-1} n^{\prime}\right)$, and $R_{n}(f)\left(n^{\prime}\right)=f\left(n^{\prime} n\right)$. These formulas reduce to the more familiar integration formulas if $D$ is also in $\mathscr{S}(N)$, or more properly, if $D=D_{f}$, where $D_{f}(g)=$ $\int_{N} f(n) g(n) d n$, where $f \in \mathscr{S}(N)$.

Suppose $h$ is a smooth function on $\mathfrak{R}^{*}$, such that all derivatives of $h$ grow at most polynomially. We may regard (integration against) $h$ as a tempered distribution on $\mathfrak{R}^{*}$. Thus the Fourier transform $\hat{h}$ of $h$ exists as a tempered distribution on $\mathfrak{N}$, and $\log _{*}(\hat{h})$, the pushforward of $\hat{h}$ by log, is a tempered distribution on $N$.

THeOREM 3.6. (a) If $h$ is a smooth $\mathrm{Ad}^{*} N$-invariant function on $\hat{\Re}$, with derivatives of polynomial growth, then convolution with $\log _{*}(\hat{h})$ defines a continuous endomorphism $\gamma(h)$ of $\mathscr{S}(N)$ commuting with both left and right translations.

(b) The map $h \rightarrow \gamma(h)$ is an homomorphism from the algebra of such functions (under pointwise multiplication) to the endomorphisms $\mathscr{S}(N)$. That is $\gamma\left(h_{1}\right) \gamma\left(h_{2}\right)=\gamma\left(h_{1} h_{2}\right)$.

(c) If $h$ is bounded, then $\gamma(h)$ extends to a bounded operator on $L^{2}(N)$, with norm equal to the supremum of $h$. Further, the map is $a^{*}$-homomorphism in the sense that $\gamma(h)$ is self-adjoint if and only if $h$ is real, and $\gamma(h)$ is positive if and only if $h$ is.

(d) The map $h \rightarrow \gamma(h)$ extends to an isomorphism of the $W^{*}$ algebra of bounded measurable $\mathrm{Ad}^{*} N$-invariant functions onto the von Neumann algebra on $L^{2}(N)$ commuting with both left and right translations. 
REMARKS. (a) Again, this is an integrated version of one of Kirillov's original results [8]. It is also suggested by the theory for finite groups [6], where it is an immediate consequence of the character theory.

(b) Since the $\gamma(h)$ act on $\mathscr{S}(N)$, they also act on $\mathscr{S}^{*}(N)$, and commute with the action of $N$ there. The spaces $\mathscr{S}(N / M, \psi)$ can be viewed as subspaces of $\mathscr{S}^{*}(N)$, specified by certain covariance conditions. Hence $\gamma(f)$ will preserve $\mathscr{S}(N / M, \psi)$ and thus will act on $\operatorname{ind}_{M}^{N} \psi$. Similarly, if $\Gamma \subseteq N$ is a discrete cocompact subgroup, then $\gamma(f)$ will act on $C^{\infty}(N / \Gamma)$. Thus the $\gamma(f)$ can be used to decompose these representations. The analogue of $(d)$ of the theorem will probably fail here though, since the $\gamma(f)$ can only separate the orbits "in general position." This raises the question of how to extend the $\gamma$-calculus, which one might call the "smooth central functional calculus" on $N$, to cope with the "singular representations" of $N$, when these occur.

Proof. The proof proceeds by inducction and is quite analogous to Schiffman's argument in [15]. One first breaks up everthing into subspaces transforming under characters of $\mathscr{Z}(N)$, and then one observes that a distribution which is conjugation invariant and transforms according th a given faithful character of $\mathscr{F}(N)$ actually lives on a proper normal subgroup (unless of course $N$ is abelian).

We will focus on (a) which is the most delicate part of the theorem.

Suppose $h$ is a smooth function on $\mathscr{N}^{*}$ such that all derivatives of $h$ grow at most polynomially. We may regard (integration against) $h$ as a tempered distribution on $\mathscr{N}^{*}$. Thus the Fourier transform $\hat{h}$ of $h$ exists as a tempered distribution on $\mathscr{N}$, and $\log _{*}(\hat{h})$, the pushforward of $\hat{h}$ by $\log$, is a tempered distribution on $N$. Thus we can convolve $\log _{*}(\hat{h})$ with $f \in \mathscr{S}(N)$. The result $\varphi=\log _{*}(\hat{h}) * f$ will clearly be a smooth function. I claim that for any $n \in N, \varphi(n z)$ will be rapidly decreasing as a function of $z \in \mathscr{Z}(N)$. This may be seen as follows. Let $p$ be any polynomial on $\mathscr{N}$, and let $\Delta$ be the Laplacian with respect to some basis of $\mathscr{N}$. Then $(p h)^{\wedge-1}=\left(p^{\wedge-1}\right)(h)$ is a sum of partial derivatives of $h$, while $\hat{\Delta}=\hat{\Delta}^{-1}$ is a negative definite quadratic form on $\mathscr{N}^{*}$, so that $(1-\hat{\Delta})^{-k}(p h)^{\wedge-1}$ will be a continuous integrable function on $\mathscr{N}^{*}$ for $k$ large enough. Hence we may write

$$
\hat{h}(f)=\int_{\mathscr{r}} g(n)\left(1-\Delta^{k}\right)(f) d n
$$

where $g$ is a continuous function such that $g p$ is bounded. Therefore for $n \in N$ and $z \in \mathscr{L}(N)$ we have 


$$
\begin{aligned}
\log _{*}(h) * f(n z) & =\log _{*}(h)\left(L_{z} L_{n}(\check{f})\right) \\
& =\int_{\mathscr{r}} g(m)(1-\Delta)_{k}\left(L_{z}\left(L_{n} \check{f}\right) \exp m\right) d m \\
& =\int_{\mathscr{r}} g(m)(1-\Delta)^{k}\left(L_{n} \check{f}\right)(\exp (m-\log z)) d m .
\end{aligned}
$$

Since for large $k$, the function $g$ will decrease faster than a given polynomial, and for fixed $k$, the function $\left(L_{n} \breve{f}\right)(\exp (m-\log z))$ is rapidly decreasing as a function of $\log z$, our claim follows.

Now for $f \in \mathscr{S}(N)$, or more generally if $f(n z)$ is in $\mathscr{S}(\mathscr{Z}(N))$ for every $n \in N$, we may define, for $\chi \in \mathscr{Z}(N)^{\wedge}$,

$$
\hat{f}(n ; \chi)=\int_{\mathscr{x}(N)} f(n z) \overline{\chi(z)} d z \text {. }
$$

Then one has

$$
\hat{f}(n z ; \chi)=\chi(z) \hat{f}(n ; \chi)
$$

or in other words $f \in \mathscr{S}(N / \mathscr{\varkappa}(N), \chi)$, and

$$
f(n)=\int_{\mathscr{x}(N) \wedge} f(n ; \chi) d \chi
$$

for proper normalization of $d \chi$.

For each $\lambda \in \mathscr{Z}(\mathscr{N})^{*}$, let $X_{\lambda}$ in $\mathscr{N}^{*}$ be the affine subspace of functionals whose restriction to $\mathscr{Z}(\mathscr{N})$ is $\lambda$. Choices of Haar measure on $\mathscr{N}^{*}$ and on $\mathscr{L}(\mathscr{N})^{*}$ give rise to a canonical Heaar measure $d x$ on each $X_{\lambda}$. For $h$ a smooth function on $\mathscr{N}^{*}$, let $h_{\lambda}$ be the restriction of $h$ to $X_{\lambda}$. We consider $h_{\lambda}$ a distribution on $X_{\lambda}$ by multiplying it by $d x$. Standard abelian Fourier analysis shows that if $\chi(z)=e^{2 \pi i \lambda(\log z)}$ for $z \in \mathscr{Z}(N)$, then

$$
\begin{aligned}
\left.\log _{*}(\hat{h}) * f\right)(n ; \chi) & =\left(\log _{*}\left(\hat{h}_{\lambda}\right) * f\right)(n) \\
& =\left(\log _{*}\left(\hat{h}_{\lambda}\right) \approx f(; \chi)(n)\right.
\end{aligned}
$$

where $*$ indicates the natural convolution on $\mathscr{S}(N / \mathscr{Z}(N), \chi)$, (or more precisely, of $\mathscr{S}^{*}(N / \mathscr{F}(N), \chi)$ with $\left.\mathscr{S}(N / \mathscr{Z}(N), \chi)\right)$. Since we know $\log _{*}(h) * f$ is rapidly decreasing in the direction of $\mathscr{z}(N)$, it will sufffice to prove that for $h$ as in the statement of the theorem, that $\log _{*}\left(\hat{h}_{\lambda}\right) \tilde{*} f(; \chi)$ is in $\mathscr{S}(N / \mathscr{Z}(N) ; \chi)$, with some sort of uniformity in $\chi$. Note that the estimate given above for the decrease of $\log _{*}(h) * f$ in the direction of $\mathscr{\digamma}(N)$ depended only on the rate of growth of the derivatives of $h$. Similarly, the estimates given below for $\log _{*}\left(h_{\lambda}\right) * f(; \chi)$ will be seen to depend only on the derivatives of $h_{\lambda}$ and, at the last, on the group law of $N$, and to grow polynomially in $\lambda$. This will suffice.

Consider convolution in $\mathscr{S}(N / \mathscr{L}(N): \chi)$. If $Y \subseteq \mathscr{Z}(N)$ is the 
identity compoent of the kernel of $\chi$, then functions in $\mathscr{S}(N / \mathscr{F}(N), \chi)$ factor to $\mathscr{S}\left((N / Y) /(\mathscr{Z}(N) / Y), \chi_{1}\right)$ where $\chi_{1}$ denotes the factorization of $\chi$ to $\mathscr{Z}(N) / Y$. If $\mathscr{z}(N / Y)$ strictly contains $\mathscr{Z}(N) / Y$, then we may break $\mathscr{S}(N / \mathscr{Z}(N), \chi)$ into smaller pieces, transforming according to the characters of $\mathscr{Z}(N / Y)$. In this case we may assume the desired estimates are true by induction on $\operatorname{dim} N$. Thus the crucial case is when $\mathscr{Z}(N / Y)=\mathscr{Z}(N) / Y$. In this case we may as well take $Y=\{0\}$, so $\operatorname{dim} \mathscr{Z}(N)$ is one-dimensional, the well-known situation. Up to this point, we have not used the assumption that $h$ was $\mathrm{Ad}^{*} N$-invariant. Our reasoning applied to any $h$. But now if $h$ is $\mathrm{Ad}^{*} N$-invariant, it must be constant on cosets of $C\left(\mathscr{Z}^{(2)}(\mathscr{N})\right)^{\perp}$, where $C\left(\mathscr{L}^{(2)}(\mathscr{N})\right.$ is the centralizer of the second center of $\mathscr{N}$. Therefore $\hat{h}$ or $\hat{h}_{\lambda}$ is in the image of the inclusion map

$$
i_{*}: \mathscr{S}^{*}\left(C\left(\mathscr{Z}^{(2)}(\mathscr{N})\right) \longrightarrow \mathscr{S}^{*}(\mathscr{N})\right. \text {. }
$$

By induction, we may assume convolution with $\hat{h}_{\lambda}$ is a continuous endomorphism of $\mathscr{S}^{*}\left(C\left(\mathscr{Z}^{(2)}(N) / \mathscr{F}(N), \chi\right)\right.$ with estimates depending only on the derivatives of $h_{\lambda}$ and the group law of $C\left(\mathcal{F}^{(2)}(N)\right)$ and with polynomial dependence on $\lambda$. Then it is clear the analogous statement is true for $h_{\lambda}$ and $\mathscr{S}^{*}(N / Z(N), \chi)$.

The other statements of the theorem can easily be proven by the same argument.

Thus "smeared orbital integrals" give rise to the central decomposition of $\mathscr{S}(N)$ or of $L^{2}(N)$. Suppose $\mathcal{O}$ is an $\operatorname{Ad}^{*} N$ orbit in $\mathfrak{R}^{*}$. Let $d \mathscr{O}$ be the invariant measure on $\mathcal{O}$. We may regard $d \mathscr{O}$ as a tempered distribution on $\mathfrak{R}^{*}$ in the obvious way. Although convolution with $\log _{*}(d \hat{\mathcal{O}})$ will not be an endomorphism of $\mathscr{S}(N)$, or bounded on $L^{2}$, it is still of interest to consider it.

Recall that if $\rho$ is a unitary representation of $N$ on $\mathscr{H}$, and if $u, v \in \mathscr{H}$, then the function $\phi_{u, v}$ on $N$ given by $\phi_{u, v}(n)=(u, \rho(n) v)$ is called a (left) matrix coefficient of $\rho$. Somewhat more generally, if $T \in \mathscr{L}(\mathscr{H})$ is a trace class operator, then $\operatorname{tr} \rho(T)(n)=\operatorname{trace}\left(\rho(n)^{-1} T\right)$ is a matrix coefficient of $\rho$, and $\operatorname{tr} \rho: T \rightarrow \operatorname{tr} \rho(T)$ defines a continuous map from the trace class operators on $\mathscr{H}$ (with the trace norm) to continuous bounded functions on $N$ (with the sup norm). Now suppose $\rho$ is irreducible. Then if $T \in C^{\infty}(\mathscr{L}(\rho))$, we have seen that $T$ is a trace class. We define the image of

$$
\operatorname{tr} \rho: C^{\infty}(\mathscr{L}(\rho)) \longrightarrow C^{\infty}(N)
$$

to be the space of smooth matrix coefficients of $N$.

THEOREM 3.7. Let $\rho \in \hat{N}$, and let $\mathcal{O} \subseteq \mathfrak{N}^{*}$ be the corresponding orbit. Then left convolution by $\log _{*}(d \hat{\mathcal{O}})$ maps $\mathscr{S}(N)$ surjectively 
on the space of smooth matrix coefficients of $\rho$.

Proof. It is well-known [8] that if $d \mathcal{O}$ is suitably normalized, then for $f \in \mathscr{S}(N)$ we have trace $\rho(f)=\log _{*}(d \hat{\mathcal{O}}) * f(1)$. The theorem follows quickly from Theorem 3.4 and this fact.

In view of this result and the motivating discussion in $\S 1$, it becomes of interest to know the behavior of $d \hat{0}$. In the nonabelian nilpotent case, $d \hat{\mathcal{O}}$ is never a function-it is always supported on some proper normal subgroup. Indeed, following Corwin and Greenleaf [4], and Penney [12], we define the saturation of $\mathscr{O}$ to be the set $s(\mathcal{O})=\left\{\lambda \in \mathfrak{R}^{*}: \lambda+\mathscr{O}=\mathcal{O}\right\}$. It is not hard to show that $s(\mathcal{O})$ is the orthogonal complement of the ideal in $\mathfrak{R}$ generated by the radicals of $B_{\lambda}, \lambda \in \mathcal{O}$. Call this ideal $\mathfrak{S}_{1}(\rho)$. The next result is clear.

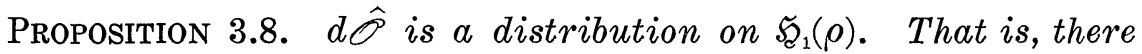
is a distribution $(d \hat{\mathcal{O}})_{1}$ on $\mathfrak{S}_{1}(\rho)$ such that $d \hat{\mathcal{O}}(f)=(d \hat{\mathcal{O}})_{1}\left(f_{\mid \tilde{q}_{1}}\right)$ for $f \in \mathscr{S}(N)$.

One can ask for much more. One can ask for the precise support of $d \hat{\mathscr{O}}$ and what sense, if any, the "values" or the "size" of $d \hat{O}$ has. In this connection, recent results of Penney [12] should be helpful.

Even the above crude result helps us to focus on the behavior of the matrix coefficients. To this end, we define the wave front set of an irreducible representation $\rho$ of $N$. If $n \in N$, we will say $n$ is in $W F(\rho)$, the wave front set of $\rho$, if we can not find a neighborhood $U$ of $\log n$ in $\mathfrak{N}$ such that for any smooth matrix coefficient $\phi$ of $\rho$, we have $|\phi(\exp t u)| \leqq c_{k, \phi}(1+|t|)^{-k}$ for any $u \in U$ and $k \in \boldsymbol{Z}$. Clearly $W f(\rho)$ is a closed set.

Proposition 3.9. (a) For $\rho \in \hat{N}$, the set $W F(\rho)$ is contained in $H_{1}(\rho)$. In particular, smooth matrix coefficients of $\rho$ die rapidly (faster than any polynomial) in any direction not in $H_{1}(\rho)$.

(b) Suppose $\rho \in \hat{N}$ is locally faithful. Then $\rho$ is square integrable if and only if $W F(\rho)=\mathscr{Z}(N)$. In that case, the smooth matrix coefficients are precisely $\mathscr{S}(N / \mathscr{Z}(N), \chi)$ where $\chi$ is the character of $\mathscr{Z}(N)$ corresponding to $\rho$.

Proof. Statement (a) follows quickly from 3.7 and 3.8. For (b), it is clear that if $W F(\rho)=\mathscr{z}(N)$, then matrix coefficients of $\rho$ vanish rapidly in any noncentral direction, so are square-integrable modulo $\mathscr{Z}(N)$. Conversely, from our discussion of square-integrable representations, we know $H_{1}(\rho)=\mathscr{z}(N)$, and in fact, $d \hat{\mathscr{O}}$ is just $\chi$ 
times Haar measure on $\mathscr{Z}(N)$. This clearly gives the rest of (b) using 3.7.

Thus we see smooth matrix coefficients vanish rapidly in almost all directions, but they must decay slowly in certain noncentral directions unless $\rho$ is square-integrable. Also note in the situation of $3.9(\mathrm{~b})$, the space $\mathscr{S}(N / \mathscr{Z}(N), \chi)$ is a simple topological algebra under convolution. Moreover, $\rho$ defines an isometry from $L^{2}(N / \mathscr{Z}(N), \chi)$ to the Hilbert-Schmidt operators on the space of $\rho$.

4. Return to the consideration of the oscillatory integrals $E_{p}$ defined in $\S 1$ (1). It is known that there is an asymptotic expansion [1], [2], [3], [10]

$$
E_{t p}(f) \sim \Sigma c_{a, k} e^{-l k / 2+a} \log ^{k} t,
$$

with $0 \leqq k \leqq n-1$, and $\alpha \in \boldsymbol{Q}$ belonging to one of a finite number of arithmetical progressions. I would like to address here the issue of why such an expansion ought to exist.

As in $\S 1$, let $A$ denote the translation group on $L^{2}(\boldsymbol{R})$, let $\mathfrak{D}$ be the space of operators of multiplication by $q$, where $q$ is a linear combination of $p$ and its partial derivatives of all orders and linear functions, and let $D$ be the unitary group of multiplication operators $U_{q}=e^{\prime q}$, with $q \in \mathfrak{D}$. Put $N=A \times_{s} D$.

Let $\mathfrak{D}_{1} \cong D$ be the subspace generated by linear functions and all proper partial derivatives of $p$. Assuming $p$ is not itself linear, we see that $\mathfrak{D}_{1}$ is of codimension one in $\mathfrak{D}$, and $\mathfrak{D}=\mathfrak{D}_{1} \oplus \boldsymbol{R}_{p}$. Let $D_{1} \subseteq D$ be the subgroup of $D$ corresponding to $D_{1}$, and let $N_{1}=$ $A \mathrm{X}_{s} D_{1}$. Then $N_{1}$ is a normal subgroup of codimension one in $N$. Thus $e^{i t p}=U_{t p}$ will form a one-parameter group of automorphisms of $N_{1}$. We note that $N_{1}$ still acts irreducibly on $L^{2}\left(\boldsymbol{R}^{n}\right)$, and that conjugation by $U_{t p}$ quite obviously leaves the associated point of $\hat{N}_{1}$ invariant.

Let $\rho$ be the representation of $N$ defined by its action on $L^{2}\left(\boldsymbol{R}^{n}\right)$, and let $\rho_{1}$ be the restriction of $\rho$ to $N_{1}$. If $\psi$ is the unitary character on $D$ such that

$$
\psi\left(e^{i q}\right)=e^{2 \pi i q(0)}
$$

then we may write $\rho=\operatorname{ind}_{D}^{N} \psi$. Thus $(N, D, \psi)$ is a normal pclarizing triple for $\rho$. Similarly, if $\psi_{1}=\psi_{\mid D_{1}}$, then $\left(N_{1}, D_{1}, \psi_{1}\right)$ is a normal polarizing triple for $\rho_{1}$.

As we remarked in $\S 1$, Haar measure on $\boldsymbol{R}^{n}$ may be interpreted as the unique linear functional on $\mathscr{S}\left(\boldsymbol{R}^{n}\right)=C^{\infty}(\rho)$ which is invariant by $\rho^{*}(A)$. (Here $\rho^{*}$ indicates the action on $C^{\infty *}(\rho)$ contragredient to $\rho$.) The appropriate group theoretic formulation of this fact is as follows. 
LEMmA 4.1. Let $M_{1}$ and $M_{2}$ be subgroups of the nilpotent group $M$ such that $M=M_{1} M_{2}$. Then for unitary characters $\psi_{i}$ of $M_{i}$, there is up to multiples at most one distribution $\lambda$ on $\mathscr{S}\left(N / M_{2} ; \psi_{2}\right)$ such that $\lambda$ is an eigendistribution with eigencharacter $\psi_{1}$ under the left action of $M_{1}$. A nontrivial such $\lambda$ exists if and only if $\psi_{1}$ and $\psi_{2}$ agree on $M_{1} \cap M_{2}$.

Proof. This is obvious.

Going further in our discussion in $\S 1$, we noted that the oscillatory integrals $E_{t p}$, defined by (1) of $\S 1$ are invariant for $\rho\left(\operatorname{Ad} U_{t p}(A)\right)$, and are characterized up to multiples by this property. Since the $E_{t p}$ are defined only up to multiples, it is appropriate to regard them as points in $\boldsymbol{P} C^{\infty *}(\rho)$, the projectivized dual of $C^{\infty}(\rho)$ (concretely, the projectivized tempered distributions). Thus we have a situation where $A_{t}=\operatorname{Ad} U_{t p}(A)$ is moving inside $N_{1}$, (or $\mathfrak{A}_{t}$ is moving inside $\mathfrak{R}_{1}$ ) and simultaneously $E_{t p}$ is moving inside $\boldsymbol{P} C^{\infty *}(\rho)$. As $t \rightarrow \infty$, we know $\mathfrak{A}_{t}$ will approach some limiting subalgebra $\mathfrak{B} \subseteq$ $\mathfrak{N}_{1}$. If the $E_{t p}$ approach some limiting point in $\boldsymbol{P} C^{\infty *}(\rho)$, then this distribution will evidently be invariant for $\rho(B)$. The philosophy we would like to adopt is that the map $A_{t} \rightarrow E_{t p}$ is actually the restriction to some open set (an "affine model") of a continuous, or even smooth, map from some projective variety $X$ of subalgebras of $\mathfrak{N}_{1}$ to (projectived) distributions invariant by the corresponding subgroups. This hyperplane section bundle on $P C^{\infty *}(\rho)$ would then induce a smooth line bundle over $X$, and the evaluation map would map $C^{\infty}(\rho)$ into smooth sections of this bundle. From this point of view, an asymptotic expansion of the form (1) would then simply be a Taylor's series for these smooth sections with respect to appropriate coordinates.

This philosophy works quite precisely when $p$ is an homogeneous quadratic polynomial. In that case, $N_{1}$ is an Heisenberg group, and the $E_{t p},-\infty<t<\infty$ together with the Dirac $\delta$ at the origin form a projective line inside $P C^{\infty *}(\rho)$. The standard proof [5] of the asymptotic expansion (5) of $\S 1$ may be interpreted in this light, or an alternative proof, based directly on the group theory, may be given.

As might be expected, a direct attempt to apply this philosophy to general polynomials runs into trouble. Computations reveal the following facts.

LEMMA 4.2. Let notations be as in the above discussion.

(i) If the partial derivatives $\partial p / \partial x_{i}$ are linearly independent, then $\mathfrak{B}$ is their linear span. 
For (ii) and (iii) assume (i) holds.

(ii) The support of any distribution invariant under $B$ is contained in the common zeros of $\partial p / \partial x_{i}$ (the singular points of $(p)$ ).

(iii) If $p$ has an isolated singularity at 0 , then the B-invariant distributions are precisely those combinations of partial derivatives at 0 which annihilate the ideal in the formal power series generated by the $\partial p / \partial x_{i}$.

From the final fact, we see that $B$ will have an essentially unique invariant distribution precisely when the $\partial p / \partial x_{i}$ generate all formal power series with zero constant term, that is, when $p$ is a nondegenerate or Morse singularity, or essentially when $p$ is a quadratic form, that is, $N_{1}$ is Heisenberg. Thus we must skirt the issue in some way. We will retreat to a more abstract generalization of the Heisenberg group, where our ideal conditions hold. We will then attempt to relate this situation to the more concrete one which motivated us.

Let $N$ now denote a nilpotent group with a locally faithful square-integrable representation $\rho$. Let $\chi$ be the character of $\mathscr{Z}(N)$ defined by $\rho$. Let $\lambda \in \mathfrak{R}$ be a point on the orbit corresponding to $\rho$. This orbit will then be $\lambda+\mathscr{Z}(\mathfrak{R})^{\perp}$. Let $V \cong \mathfrak{N}$ be the kernel of $\lambda$. Suppose $(\mathfrak{N}, \mathfrak{M}, \lambda)$ is a polarizing triple for $\rho$. If $\mathfrak{M}_{0}=\mathfrak{M} \cap V$, then we see $\mathfrak{M}_{0}=\operatorname{ker} \lambda_{\mid \mathfrak{M}}$ and $\mathfrak{M}=\mathfrak{M}_{0} \oplus \mathscr{Z}(\mathfrak{N})$. Also it is not hard to see that $\mathfrak{M}_{0}$ is an isotropic subspace of maximal dimension in $V$ with respect to $B_{\lambda}$, and that $\mathfrak{M}_{0}$ is a subalgebra of $\mathfrak{R}$. Conversely, if $\mathfrak{M}_{0} \cong V$ is a subspace enjoying these two properties, then putting $\mathfrak{M}=\mathfrak{M}_{0} \oplus \mathscr{L}(\mathfrak{R})$, we see $(\mathfrak{N}, \mathfrak{M}, \lambda)$ is a polarizing triple for $\rho$. In summary, we conclude polarizing triples $(\mathfrak{N}, \mathfrak{M}, \lambda)$ are in bijective correspondence with maximal $B_{\lambda}$-isotropic subspaces of $V$ which are

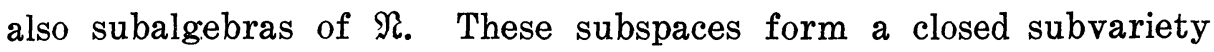
of the flag manifold of maximal $B_{R}$-isotropic subspaces of $V$. Call this subvariety $\Pi$. Over $\Pi$ there is a natural vector bundle $C$ which attaches to each point in $\Pi$ the subspace of $V$ which defines it. If $\operatorname{dim} V=2 n$, then elements of $\Pi$ have dimension $n$. Thus $\Lambda^{n}(C)$, the $n$th exterior power of $C$, is a line bundle over $\Pi$, called the volume bundle of $\Pi$.

According to Corollary 3.4.3, for each $\mathfrak{M}_{0} \in \Pi$, there is on $C^{\infty}(\rho)$ a unique (up to multiples) linear functional $\lambda\left(\mathfrak{M}_{0}\right)$ which is invariant under $\rho^{*}\left(M_{0}\right)$. Thus we may define a map

$$
\lambda: \Pi \longrightarrow P C^{\infty *}(\rho)
$$

by $\lambda: \mathfrak{M}_{0} \rightarrow \lambda\left(\mathfrak{M}_{0}\right)$. Let $\Lambda$ denote the pullback by $\lambda$ of the hyperplane section bundle on $\boldsymbol{P} C^{\infty *}(\rho)$. This $\Lambda$ is a complex line bundle over $\Pi$. 
THEOREM 4.3. (a) The map $\lambda$ is smooth, in the sense that if $f:[0,1] \rightarrow \Pi$ is a smooth curve, then $f^{*}(\Lambda)$ is a smooth vector bundle over $[0,1]$, and sections of $f^{*}(\Lambda)$ obtained by pulling back sections of the hyperplane section bundle are smooth sections.

(b) If $\bar{\Lambda}$ is the complex conjugate of $\Lambda$, then $\Lambda \otimes \bar{\Lambda}$ is the (complexification of) the volume bundle of $\Pi$.

REMARK. When $N$ is the Heisenberg group, this result is due to Kostant [9].

Proof. Given $\mathfrak{M}_{0} \in \Pi$, we define on $\mathscr{S}(N / \mathscr{L}(N), \bar{\chi})$ (where $\bar{\chi}$ is the complex conjugate of $\chi)$ a distribution $\nu\left(M_{0}\right)=\nu$ by the formula

$$
\nu(f)=\int_{M_{0}} f(m) d m,
$$

where $d m$ is Haar measure on $M_{0}$. We will abbreviate $\mathscr{S}(N / \mathscr{Z}(N), \bar{\chi})=$ $\mathscr{S}$. It is easy to see that $\nu: \Pi \rightarrow \mathscr{S}^{*}$ is smooth, and that the associated line bundle over $\Pi$ is just the volume bundle. Moreover, $\nu\left(M_{0}\right)$ is both right and left invariant. Since $L^{2}(N / \mathscr{2}(N), \bar{\chi})$ forms an irreducible $N \times N$ module under right and left translation, this module being isomorphic to $\rho \otimes \rho^{*}$ (outer tensor product), another application of Corollary 3.4.3 shows $\nu\left(M_{0}\right)$ is essentially the only left and right $M_{0}$-invariant distribution on $\mathscr{S}$. Moreover, by Proposition 3.2, left or right convolution with $\nu\left(M_{0}\right)$ projects $\mathscr{S}$ onto $\mathscr{S}\left(N / M, \bar{\chi}_{M}\right)$, where $M=M_{0} \mathscr{Z}(N)$ and $\bar{\chi}_{M}$ agrees with $\bar{\chi}$ on $\mathscr{Z}(N)$ and is trivial on $M_{0}$.

Take a vector $v \in C^{\infty}(\rho)$ and define $\Phi: C^{\infty}(\rho) \rightarrow \mathscr{S}$ by $\Phi(w)(n)=$ $(u, \rho(n) v)$. The usual theory of square-integrable representations says $\Phi$ is isometric with respect to the appropriate inner products, and embeds the space of $\rho$ as a submodule of $\mathscr{S}$ under the left action of $N$. Moreover, $\Phi(v)$ is an idempotent in $\mathscr{S}$, and the image of $\Phi$ is precisely $\mathscr{S} * \Phi(v)$. It follows that the unique $\rho^{*}\left(M_{0}\right)$-invariant distribution is precisely $\nu\left(M_{0}\right) * \mathscr{S} * \Phi(v)$. It is evident that this depends smoothly on $\mathfrak{M}_{0}$. This proves (a). For (b), note that $\lambda\left(\mathfrak{M}_{0}\right) \otimes \bar{\lambda}\left(\mathfrak{M}_{0}\right) \in C^{\infty *}(\rho) \otimes C^{\infty *}(\bar{\rho}) \cong C^{\infty *}(\mathscr{L}(\rho)) \cong \mathscr{S}^{*}$ will define an $M_{0}$ bi-invariant distribution on $\mathscr{S}$, which must therefore be $\nu\left(\mathfrak{M}_{0}\right)$. This proves (b).

The remaining discussion is primarily speculative.

Return to the situation considered at the beginning of this section. In particular, reconsider the groups $N=A \times{ }_{s} D$ and $N_{1}=$ $A \times_{s} D_{1}$. We know that $\left(N_{1}, D_{1}, \psi_{1}\right)$, where $\psi_{1}$ is as defined in $\S 4$, (2), is a normal polarizing triple for a locally faithful representation $\rho_{1}$ of $N_{1}$. In particular, $\left(N_{1}, D_{1}\right)$ is a maximal normal abelian $n$-pair. If $\left(\mathfrak{D}_{1}, S_{0}, \mathfrak{A}, \alpha\right)$ is the associative correlative of $\left(N_{1}, D_{1}\right)$, then $\alpha=0$ 
since $N_{1}$ is a semidirect product. The algebra $S_{0}$ is the algebra of constant coefficient differential operators restricted to $\mathfrak{D}_{1}$, and $\mathfrak{A}$ is the subspace first-order operators. We put of $\tilde{\mathfrak{R}}_{1}=S_{0} \mathrm{X}_{s} \mathfrak{D}_{1}$. One easily checks that $\tilde{N}_{1}$ contains $N_{1}$ as a normal subgroup, and that $\left(\widetilde{N}_{1}, D_{1}, \psi_{1}\right)$ is a normal polarizing triple for an irreducible squareintegrable representation of $\tilde{N}_{1}$. It follows that $\tilde{N}_{1}$ is the split HAT group associated to $S_{0}$.

We may perform the same construction with $\mathfrak{R}_{1}$ replaced by $\mathfrak{N}$. Let the associative correlative of $\left(\mathfrak{N}\right.$, () be $\left(\mathfrak{D}, T_{0}, \mathfrak{A}, 0\right)$. Note that $S_{0}$ is the quotient of $T_{0}$ by the one-dimensional ideal $Z$ in $T_{0}$ which annihilates $\mathfrak{D}_{1}$. Define $\tilde{\mathfrak{R}}=T_{0} \mathrm{X}_{8} \mathfrak{D}$. Then $\tilde{N}$ is again an HAT group, and the inclusion $\mathfrak{D}_{1} \subseteq \mathfrak{D}$ can be extended to an embedding $\widetilde{N}_{1} \hookrightarrow \widetilde{N}$ as normal subgroup. In fact, $\mathscr{D}_{1}$ is the commutator ideal in $\tilde{R}$, and its centralizer is $Z+\mathfrak{D}$. Choosing an embedding $\tilde{N}_{1} \rightarrow \tilde{N}$ amounts to choosing a complement to $Z$ in $T_{0}$. Also, it results in a decomposition of the type described in Proposition 2.6(c). Recall that our polynomial $p$ defines a complement to $\mathfrak{D}_{1}$ in $\mathfrak{D}$. Choosing: this complement defines, in the manner of 2.6(c), a complement $S_{0}^{\prime}$ to $Z$ in $T_{0}$. We may use $S_{0}^{\prime}$ to define a standard embedding $\widetilde{N}_{1} \rightarrow \widetilde{N}$ as normal subgroup. Then the line through $p$ in $\tilde{\mathfrak{R}}$ will define outer derivations of $\widetilde{\Re}_{1}$, which may be exponentiated (actually, their squares are already zero) to give a one-parameter group of outer automorphisms, exp ad $t p=\alpha_{t}$.

Let $\widetilde{A}=1+S_{0} \subseteq \widetilde{N}_{1}$. Then $\widetilde{A} \mathscr{Z}\left(\widetilde{N}_{1}\right)$ is a polarizing subgroup for $\tilde{\rho}_{1}$. Therefore there is in $C^{\infty *}\left(\tilde{\rho}_{1}\right)$ an essentially unique point fixed by $\tilde{A}$. Let $\alpha_{t}$ act on $\tilde{N}_{1}$. Put $\alpha_{t}(\widetilde{A})=\tilde{A}_{t}$. As $t \rightarrow \infty$, the groups $\widetilde{A}_{t}$ will approach a limiting position $\widetilde{B}$. According to Theorem 4.3 , the distributions corresponding to the $\widetilde{A}_{t}$ must approach in $P C^{\infty *}\left(\tilde{\rho}_{1}\right)$ the distribution corresponding to $\widetilde{B}$.

How does that look concretely? We may realize $\tilde{\rho}_{1}$, analogously to $\rho_{1}$, as $\operatorname{ind}_{D_{1}}^{\tilde{N}_{1}} \psi_{1}$. Further, we may identify the space of $\tilde{\rho}_{1}$ with $L^{2}(\widetilde{A})$, and then $\widetilde{A}$ will act by translations and $D_{1}$ will act by multiplicaton by exponential polynomials in appropriate coordinates. (In fact, coordinates on $\tilde{A}$ may be introduced so that $D_{1}$ will act by exponentials of linear polynomials.) In any case, the automorphisms $\alpha_{t}$ will preserve $\tilde{\rho}_{1}$, so that we may extend $\tilde{\rho}_{1}$ to the group $\alpha_{t}$. In this realization $\tilde{\rho}_{1}\left(\alpha_{t}\right)$ will also act as multiplication by an exponential polynomial. This polynomial on $\widetilde{A}$ will be closely related to the socalled "universal unfolding" of $p$ [16]. For this reason, it seems not too far-fetched to call $\tilde{\rho}_{1}$ the universal unfolding of $\rho_{1}$.

Hopefully the construction of $\tilde{\rho}_{1}$ affords some sense of why asymptotic expansions of the type of $\S 4$ (1) should exist. However, something is certainly lost in the process: our original translation group $A$ is now only a small subgroup of $\tilde{A}$, and $\mathscr{S}(A)$ is not a 
subspace of $\mathscr{S}(\tilde{A})$, but must be regarded only as a subspace of $\mathscr{S}^{*}(\widetilde{A})$. Thus the translation of the asymptotic expansion given by Theorem 4.3 into an expansion of the type of $\S 4$ (1) presents technical difficulties. These difficulties are quite possibly no less considerable than the existing proofs of $\S 4$ (1). Thus it is moot at this point whether Theorem 4.3 can be translated into concrete formulas concerning $E_{t p}$. The answer will require further work.

Finally, we remark that the question of the Fourier transform of $e^{i t p}$ is connected with the possibility of realizing $\tilde{\rho}_{1}$ also as being induced from $\widetilde{A} \cdot \mathscr{Z}\left(\tilde{N}_{1}\right)$, for the intertwining operator between the two realizations is essentially the Fourier transform.

\section{REFERENCES}

1. M. F. Atiyah, Resolution of singularities and division of distributions, Comm. Pure and App. Math., 23 (1970).

2. I. N. Bernstein, The analytic continuation of generalized functions with respect to a parameter, Funk. Anal. Pril., 6 (1972), no. 47, pp. 26-40.

3. I. N. Bernstein and S. I. Gelfand, The function $P^{\lambda}$ is meromorphic, Funk. Anal. Pril., 3 (1967), no. 1, pp. 84-86.

4. L. Corwin and F. Greenleaf, in preparation.

5. J. J. Duistermaat, Fourier Integral Operators, Courant Institute Lecture Notes, N. Y. U., New York, 1973.

6. R. Howe, On representations of discrete, finitely generated, torsion-free nilpotent groups, Pacific J. Math., 73 (1977), 281-305.

7. —, Topics in harmonic analysis on solvable algebraic groups, Pacific J. Math., 73 (1977), 383-435.

8. A. A. Kirillov, Unitary representations of nilpotent Lie groups, Usp. Mat. Nauk., 17 (1962), 57-110.

9. B. Kostant, Symplectic spinors, Symp. Math. V, 14 (1974), 139-152.

10. B. Malgrange, Sur les polynomes de I. N. Bernstein, Usp. Mat. Nauk., 29 (1974), no. $4,178$.

11. C. C. Moore and J. Wolfe, Square integrable representations of nilpotent groups, Trans. Amer. Math. Soc., 185 (1974), 445-462.

12. R. Penney, Canonical objects in the Kirillov theory of nilpotent Lie groups, to appear P.A.M.S.

13. N. S. Poulsen, On $C^{\infty}$-vectors and intertwining bilinear forms for representations of Lie groups, J. Functional Analysis, 9 (1972), 87-120.

14. L. Pukanszky, On the theory of exponential groups, T.A.M.S., 126 (1967), 457-507.

15. G. Schiffman, Distributions centrales de type positif sur un groupe de Lie nilpotent, Bull. Soc. Math. France, 96 (1968), 347-355.

16. R. Thom, Structural Stability and Morphogenesis. W. A. Benjamin, Reading, Mass., 1975.

Received May 21, 1977.

YALE UNIVERSITY

New Haven, CT 06520 



\title{
PACIFIC JOURNAL OF MATHEMATICS
}

\section{EDITORS}

\author{
RICHARD ARENS (Managing Editor) \\ University of California \\ Los Angeles, CA 90024 \\ Charles W. Curtis \\ University of Oregon \\ Eugene, OR 97403 \\ C. C. MOORE \\ University of California \\ Berkeley, CA 94720
}

\author{
J. DUGUNDJI \\ Department of Mathematics \\ University of Southern California \\ Los Angeles, CA 90007
}

R. Finn and J. Milgram

Stanford University

Stanford, CA 94305

\section{ASSOCIATE EDITORS}

E. F. BeCKenBaCH

B. H. NeumanN

F. WOLF

K. YoSHIDA

\section{SUPPORTING INSTITUTIONS}

\author{
UNIVERSITY OF BRITISH COLUMBIA \\ CALIFORNIA INSTITUTE OF TECHNOLOGY \\ UNIVERSITY OF CALIFORNIA \\ MONTANA STATE UNIVERSITY \\ UNIVERSITY OF NEVADA, RENO \\ NEW MEXICO STATE UNIVERSITY \\ OREGON STATE UNIVERSITY \\ UNIVERSITY OF OREGON
}

\author{
OSAKA UNIVERSITY \\ UNIVERSITY OF SOUTHERN CALIFORNIA \\ STANFORD UNIVERSITY \\ UNIVERSITY OF HAWAII \\ UNIVERSITY OF TOKYO \\ UNIVERSITY OF UTAH \\ WASHINGTON STATE UNIVERSITY \\ UNIVERSITY OF WASHINGTON
}

The Supporting Institutions listed above contribute to the cost of publication of this Journal, but they are not owners or publishers and have no responsibility for its content or policies.

Mathematical papers intended for publication in the Pacific Journal of Mathematics should be in typed form or offset-reproduced, (not dittoed), double spaced with large margins. Please do not use built up fractions in the text of the manuscript. However, you may use them in the displayed equations. Underline Greek letters in red, German in green, and script in blue. The first paragraph or two must be capable of being used separately as a synopsis of the entire paper. Items of the bibliography should not be cited there unless absolutely necessary, in which case they must be identified by author and journal, rather than by item number. Manuscripts, in triplicate, may be sent to any one of the editors. Please classify according to the scheme of Math. Reviews, Index to Vol. 39. All other communications should be addressed to the managing editor, or Elaine Barth, University of California, Los Angeles, California, 90024.

The Pacific Journal of Mathematics expects the author's institution to pay page charges, and reserves the right to delay publication for nonpayment of charges in case of financial emergency.

50 reprints to each author are provided free for each article, only if page charges have been substantially paid. Additional copies may be obtained at cost in multiples of 50 .

The Pacific Journal of Mathematics is issued monthly as of January 1966. Regular subscription rate: $\$ 72.00$ a year (6 Vols., 12 issues). Special rate: $\$ 36.00$ a year to individual members of supporting institutions.

Subscriptions, orders for numbers issued in the last three calendar years, and changes of address should be sent to Pacific Journal of Mathematics, 103 Highland Boulevard, Berkeley, California, 94708. Older back numbers obtainable from Kraus Periodicals Co., Route 100, Millwood, NY 10546.

\footnotetext{
PUBLISHED BY PACIFIC JOURNAL OF MATHEMATICS, A NON-PROFIT CORPORATION

Printed at Kokusai Bunken Insatsusha (International Academic Printing Co., Ltd.). 8-8, 3-chome, Takadanobaba, Shinjuku-ku, Tokyo 160, Japan.
} 


\section{Pacific Journal of Mathematics}

\section{Vol. 73, No. $2 \quad$ April, 1977}

Roger Evans Howe, On representations of discrete, finitely generated, torsion-free, nilpotent groups ........................ 281

Roger Evans Howe, The Fourier transform for nilpotent locally compact

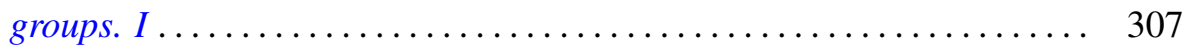

Roger Evans Howe, On a connection between nilpotent groups and

oscillatory integrals associated to singularities............... 329

Roger Evans Howe, Kirillov theory for compact p-adic groups .......... 365

Roger Evans Howe, Topics in harmonic analysis on solvable algebraic

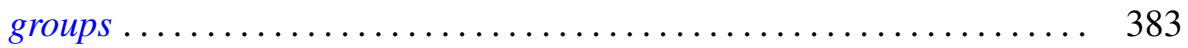

Roger Evans Howe, Tamely ramified supercuspidal representations of

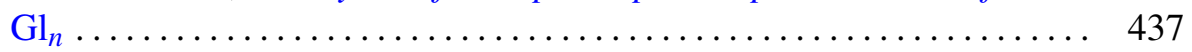

Lawrence Jay Corwin and Roger Evans Howe, Computing characters of tamely ramified $p$-adic division algebras .....................

Roger Evans Howe, Some qualitative results on the representation theory of

$\mathrm{Gl}_{n}$ over a $p$-adic field ............................. 479

Herbert Stanley Bear, Jr., Corrections to: “Ordered Gleason parts”. . . . . . 539

Andreas Blass, Corrections to: "Exact functors and measurable cardinals" .....................................

Robert M. DeVos, Corrections to: "Subsequences and rearrangements of sequences in $\mathrm{FK}$ spaces". 